\title{
STRONG CLASSIFICATION OF PURELY INFINITE CUNTZ-KRIEGER ALGEBRAS
}

\author{
TOKE MEIER CARLSEN, GUNNAR RESTORFF, AND EFREN RUIZ
}

\begin{abstract}
In 2006, Restorff completed the classification of all Cuntz-Krieger algebras with finitely many ideals (i.e., those that are purely infinite) up to stable isomorphism. He left open the questions concerning strong classification up to stable isomorphism and unital classification. In this paper, we address both questions. We show that any isomorphism between the reduced filtered $K$-theory of two Cuntz-Krieger algebras with finitely many ideals lifts to a *-isomorphism between the stabilized Cuntz-Krieger algebras. As a result, we also obtain strong unital classification.
\end{abstract}

\section{INTRODUCTION}

Historically there has been a lot of connection between classification of shifts of finite type, classification of Cuntz-Krieger algebras and classification of more general $C^{*}$-algebras. Franks made a successful classification of irreducible shifts of finite type up to flow equivalence [Fra84, which Rørdam used to classify simple, purely infinite Cuntz-Krieger algebras Ror95] using a trick of Cuntz. This was shortly after generalized by Kirchberg-Phillips to a strong classification of simple, nuclear, purely infinite $C^{*}$-algebras in the UCT-class, i.e., allowing for lifting of isomorphisms on the invariant level to the algebra level ( $c f$. Phi00, Kir00]). Huang classified shifts of finite type with finite Bowen-Franks groups up to flow equivalence and used this to classify Cuntz-Krieger algebras with finite $K_{0}$-groups Hua95. Huang also classified two component shifts of finite type up to flow equivalence and used this to classify Cuntz-Krieger algebras with exactly one ideal Hua94,Hua96. This was generalized by Rørdam to essential extensions of stable, simple, nuclear, purely infinite $C^{*}$ algebras in the UCT-class Ror97. Eilers-Restorff and Restorff-Ruiz ER06, RR07. generalized this to cover classification for all essential extensions of simple, nuclear, purely infinite $C^{*}$-algebras in the UCT-class using results of Kirchberg and Bonkat Kir00, Bon04 - allowing for strong classification in the unital and the stable cases. The work in ER06, RR07, ERR13c shows that for properly infinite $C^{*}$ algebras (or for $C^{*}$-algebras with stable weak cancellation), the key to solving the unital classification problem is to get a strong classification for the stable case.

Finally, Boyle and Huang classified general shifts of finite type up to flow equivalence Boy02, BH03. Using this, Restorff classified all purely infinite Cuntz-Krieger algebras up to stable isomorphism — with the problems of strong classification and

Received by the editors June 21, 2016 and, in revised form, October 28, 2016.

2010 Mathematics Subject Classification. Primary 46L35, 46L80; Secondary 46L55, 37B10.

Key words and phrases. Cuntz-Krieger algebras, graph $C^{*}$-algebras, $K$-theory, flow equivalence.

(C) 2017 by the authors under Creative Commons Attribution-Noncommercial 3.0 License (CC BY NC 3.0) 
unital classification left as open questions in the addendum Res06, Questions 1 and 3]. These questions will be answered in this paper.

Restorff, Meyer-Nest, and Bentmann-Köhler have shown that stable, nuclear, purely infinite $C^{*}$-algebras in the UCT-class with finite ideal lattice are classified by their filtered $K$-theory if and only if their primitive ideal spaces are so-called accordion spaces - and when there is classification, it is a strong classification Res08, MN12,BK11. In contrast, all finite ideal spaces appear as the ideal space of Cuntz-Krieger algebras. Arklint-Restorff-Ruiz ARR16 showed that there are purely infinite Cuntz-Krieger algebras with four primitive ideals for which there are automorphisms of the filtered $K$-theory that cannot be lifted to the stabilized algebra, while the work of Arklint-Restorff-Ruiz and Arklint-Bentmann-Katsura shows that all Cuntz-Krieger algebras with at most four primitive ideals are strongly classified by the reduced filtered $K$-theory - both up to stable isomorphism and up to unital isomorphism ABK14b, ARR16. This suggests that the correct invariant to strongly classify Cuntz-Krieger algebras is the reduced filtered $K$-theory.

Move equivalence and Cuntz move equivalence of graphs, Cuntz-Krieger algebras and graph $C^{*}$-algebras and their interplay have recently also been important for developing results about classification of certain nonsimple $C^{*}$-algebras that are of mixed kind (e.g. ERRS16b, ERRS16a, ERRS16c, ERR13b, ERR13a, ERR09, ET10] - since we will only focus on strong classification of purely infinite Cuntz-Krieger algebras, we will not examine this further here.

In this paper, we will strongly classify all purely infinite Cuntz-Krieger algebras up to stable isomorphism and up to unital isomorphism using the reduced filtered $K$-theory as invariant, together with a way of representing the class of the unit for the unital classification (thus providing an answer to Question 1 and a positive answer to Question 3 in the addendum in [Res06]). We will do so drawing heavily on results from Boy02, BH03, Res06, ERR13c, ERRS16b, ERRS16c, and using graph $C^{*}$-algebras instead of Cuntz-Krieger algebras will be of key importance for the proof. Along the way, we will therefore show some results in slightly greater generality than strictly needed for our main theorems - these results will be of great importance in a forthcoming paper about the geometric classification of general unital graph $C^{*}$-algebras ERRS16a.

We also note that Arklint-Bentmann-Katsura have a very nice range result characterizing the range of the reduced invariant for all purely infinite Cuntz-Krieger algebras - both with and without the class of the unit ABK14a.

Before we state the main theorems in Section 3, we introduce in Section 2 some notation and definitions needed to state the main results. In Section 3 , we state the main results of this paper, and we then explain the strategy of the proofs of these results, and introduce some notation and definitions we use in the proofs. In Section 4, we strengthen some results of [BH03], and in Section 5, we describe how edge expanding a graph gives rise to stable isomorphism of graph algebras and $\mathrm{SL}_{\mathcal{P}}$ equivalence of corresponding matrices derived from the graphs. In Section 6, we examine how row and column addition of matrices give rise to stable isomorphism of graph $C^{*}$-algebras, and describe what maps on the reduced filtered $K$-theory these stable isomorphisms induce. Finally, in Section 7 we look at how Cuntz splicing a graph gives rise to stable isomorphism of graph $C^{*}$-algebras and describe what maps on the reduced filtered $K$-theory such a stable isomorphism induces, before we give the proofs of the main theorems. 


\section{Preliminaries}

We now introduce and recall some notation and definitions needed for stating the main results of this paper. Let $A$ be an $n \times n$ matrix with entries in $\mathbb{N}_{0}$, the nonnegative integers, and let $A(i, j)$ denote the $(i, j)$ th entry of $A$. We say that $A$ is nondegenerate if all its rows and columns are nonzero.

For every nondegenerate $n \times n$ matrix $A$ with entries from $\{0,1\}$, the CuntzKrieger algebra, $\mathcal{O}_{A}$, associated with $A$ is the universal (unital) $C^{*}$-algebra generated by $n$ partial isometries $s_{1}, \ldots, s_{n}$ satisfying the relations

$$
\begin{aligned}
\mathbf{1} & =s_{1} s_{1}^{*}+\cdots+s_{n} s_{n}^{*}, \\
s_{i}^{*} s_{i} & =\sum_{j=1}^{n} A(i, j) s_{j} s_{j}^{*}, \quad \text { for all } i=1, \ldots, n .
\end{aligned}
$$

It is a well-known fact that Cuntz-Krieger algebras are unital, separable, nuclear $C^{*}$-algebras in the UCT-class. In Cun81, Cuntz defined Condition (II) for a nondegenerate matrix with entries from $\{0,1\}$. A nondegenerate matrix $A$ with entries from $\{0,1\}$ satisfies Condition (II) if and only if the Cuntz-Krieger algebra $\mathcal{O}_{A}$ is purely infinite if and only if the Cuntz-Krieger algebra $\mathcal{O}_{A}$ has finitely many ideals if and only if the Cuntz-Krieger algebra $\mathcal{O}_{A}$ has real rank zero. See Res06] and the references therein.

For a $C^{*}$-algebra $\mathfrak{A}$, we let Prim $\mathfrak{A}$ denote the primitive ideal space equipped with the usual hull-kernel topology. It is a well-known fact that for every open subset $O$ of Prim $\mathfrak{A}$ the set $\mathfrak{A}(O):=\bigcap(($ Prim $\mathfrak{A}) \backslash O)$ is a (two-sided, closed) ideal of $\mathfrak{A}$, and that this is a lattice isomorphism from the lattice of open subsets of Prim $\mathfrak{A}$ to the lattice of ideals of $\mathfrak{A}$.

If $\mathfrak{A}$ has finitely many ideals, then for every subset $S$ of Prim $\mathfrak{A}$ there exists a smallest open subset $\widetilde{S}$ of Prim $\mathfrak{A}$ that contains $S$. For convenience, we will use the notation $\widetilde{\mathfrak{p}}$ for $\widetilde{\{\mathfrak{p}\}}$, and note that $\widetilde{\partial} \mathfrak{p}:=\widetilde{\mathfrak{p}} \backslash\{\mathfrak{p}\}$ is also open. The following definition is a reformulation of [Res06, Definition 4.1]; the definition can be generalized to $C^{*}$-algebras over finite $T_{0}$-spaces (see e.g. [ARR12, ABK14b, ERRS16b].

Definition 2.1. Let $\mathfrak{A}$ be a $C^{*}$-algebra with finitely many ideals. Let

$$
\begin{aligned}
I_{0}(\mathfrak{A}):=\{\widetilde{\mathfrak{p}} \mid \mathfrak{p} \in \operatorname{Prim} \mathfrak{A}\} \cup\{\widetilde{\partial} \mathfrak{p} \mid \mathfrak{p} & \in \operatorname{Prim} \mathfrak{A}\}, \\
C(\mathfrak{A}):=\{(\mathfrak{p}, \mathfrak{q}) \in \operatorname{Prim} \mathfrak{A} \times \operatorname{Prim} \mathfrak{A} \mid & \widetilde{\mathfrak{p}} \subsetneq \widetilde{\partial} \mathfrak{q} \text { and } \\
& \left.\quad \mathfrak{p}^{\prime} \in \operatorname{Prim} \mathfrak{A} \text { such that } \widetilde{\mathfrak{p}} \subsetneq \widetilde{\mathfrak{p}^{\prime}} \subseteq \widetilde{\partial} \mathfrak{q}\right\} .
\end{aligned}
$$

The reduced filtered $K$-theory of $\mathfrak{A}, \mathrm{FK}_{\text {red }}(\mathfrak{A})$, consists of Prim $\mathfrak{A}$ and the families

$$
\left(K_{0}(\mathfrak{A}(O))\right)_{O \in I_{0}(\mathfrak{A})}, \quad\left(K_{0}(\mathfrak{A}(\widetilde{\mathfrak{p}}) / \mathfrak{A}(\widetilde{\partial} \mathfrak{p}))\right)_{\mathfrak{p} \in \operatorname{Prim} \mathfrak{A}}, \quad\left(K_{1}(\mathfrak{A}(\widetilde{\mathfrak{p}}) / \mathfrak{A}(\widetilde{\partial} \mathfrak{p}))\right)_{\mathfrak{p} \in \operatorname{Prim} \mathfrak{A}}
$$

together with the sequences

$$
K_{1}(\mathfrak{A}(\widetilde{\mathfrak{p}}) / \mathfrak{A}(\widetilde{\partial \mathfrak{p}})) \longrightarrow K_{0}(\mathfrak{A}(\widetilde{\partial \mathfrak{p}})) \longrightarrow K_{0}(\mathfrak{A}(\widetilde{\mathfrak{p}})) \longrightarrow K_{0}(\mathfrak{A}(\widetilde{\mathfrak{p}}) / \mathfrak{A}(\widetilde{\partial} \mathfrak{p}))
$$

for every $\mathfrak{p} \in \operatorname{Prim} \mathfrak{A}$ and

$$
K_{0}(\widetilde{\mathfrak{p}}) \longrightarrow K_{0}(\widetilde{\partial} \mathfrak{q})
$$


for every pair $(\mathfrak{p}, \mathfrak{q}) \in C(\mathfrak{A})$ originating from the cyclic six term exact sequences induced by the extensions $\mathfrak{A}(\widetilde{\partial} \mathfrak{p}) \hookrightarrow \mathfrak{A}(\widetilde{\mathfrak{p}}) \rightarrow \mathfrak{A}(\widetilde{\mathfrak{p}}) / \mathfrak{A}(\widetilde{\partial} \mathfrak{p})$ and $\mathfrak{A}(\widetilde{\mathfrak{p}}) \hookrightarrow \mathfrak{A}(\widetilde{\partial} \mathfrak{q}) \rightarrow$ $\mathfrak{A}(\widetilde{\partial} \mathfrak{q}) / \mathfrak{A}(\widetilde{\mathfrak{p}})$, respectively.

Let $\mathfrak{B}$ be another $C^{*}$-algebra with finitely many ideals. By a reduced filtered $K$ theory isomorphism $(\kappa, \rho)$ from $\mathrm{FK}_{\text {red }}(\mathfrak{A})$ to $\mathrm{FK}_{\text {red }}(\mathfrak{B})$ we understand isomorphisms

$$
\begin{aligned}
\rho: \operatorname{Prim} \mathfrak{A} & \rightarrow \operatorname{Prim} \mathfrak{B}, & & \text { homeomorphism, } \\
\kappa_{O}^{0, i}: K_{0}(\mathfrak{A}(O)) & \rightarrow K_{0}(\mathfrak{B}(\rho(O))), & & \text { for } O \in I_{0}(\mathfrak{A}), \\
\kappa_{\mathfrak{p}}^{0, q}: K_{0}(\mathfrak{A}(\widetilde{\mathfrak{p}}) / \mathfrak{A}(\widetilde{\partial} \mathfrak{p})) & \rightarrow K_{0}(\mathfrak{B}(\widetilde{\rho(\mathfrak{p})}) / \mathfrak{B}(\widetilde{\partial} \rho(\mathfrak{p}))), & & \text { for } \mathfrak{p} \in \operatorname{Prim} \mathfrak{A}, \\
\kappa_{\mathfrak{p}}^{1, q}: K_{1}(\mathfrak{A}(\widetilde{\mathfrak{p}}) / \mathfrak{A}(\widetilde{\partial} \mathfrak{p})) & \rightarrow K_{1}(\mathfrak{B}(\widetilde{\rho(\mathfrak{p})}) / \mathfrak{B}(\widetilde{\partial} \rho(\mathfrak{p}))), & & \text { for } \mathfrak{p} \in \operatorname{Prim} \mathfrak{A},
\end{aligned}
$$

such that all the ladders coming from sequences in $\mathrm{FK}_{\text {red }}(\mathfrak{A})$ and $\mathrm{FK}_{\text {red }}(\mathfrak{B})$ commute.

It is clear that any *-isomorphism $\Psi$ from $\mathfrak{A}$ to $\mathfrak{B}$ or from $\mathfrak{A} \otimes \mathbb{K}$ to $\mathfrak{B} \otimes \mathbb{K}$ induces in a canonical way an isomorphism from $\mathrm{FK}_{\text {red }}(\mathfrak{A})$ to $\mathrm{FK}_{\text {red }}(\mathfrak{B})$. We denote these by $\mathrm{FK}_{\text {red }}(\Psi)$ and $\mathrm{FK}_{\text {red }}^{s}(\Psi)$, respectively.

There is a nice description of the ideal lattice and the $K$-theory of all subquotients as well as of the occurring cyclic six-term exact sequences in terms of the underlying matrix (see Res06] and the references therein).

Definition 2.2. Let $\mathfrak{A}$ and $\mathfrak{B}$ be $C^{*}$-algebras with finitely many ideals and having real rank zero. By ABK14b, Lemma 8.3], every isomorphism $(\kappa, \rho)$ from $\mathrm{FK}_{\text {red }}(\mathfrak{A})$ to $\mathrm{FK}_{\text {red }}(\mathfrak{B})$ induces a unique isomorphism $\kappa_{0}: K_{0}(\mathfrak{A}) \rightarrow K_{0}(\mathfrak{B})$. If $\mathfrak{A}$ and $\mathfrak{B}$ are unital, we say the reduced filtered $K$-theory isomorphism $(\kappa, \rho)$ from $\operatorname{FK}_{\text {red }}(\mathfrak{A})$ to $\mathrm{FK}_{\text {red }}(\mathfrak{B})$ preserves the class of the unit if $\kappa_{0}\left(\left[1_{\mathfrak{A}}\right]_{0}\right)=\left[1_{\mathfrak{B}}\right]_{0}$ in $K_{0}(\mathfrak{B})$. We write $\left(\mathrm{FK}_{\text {red }}(\mathfrak{A}),\left[1_{\mathfrak{A}}\right]_{0}\right) \cong\left(\mathrm{FK}_{\text {red }}(\mathfrak{B}),\left[1_{\mathfrak{B}}\right]_{0}\right)$ whenever such an isomorphism exists.

\section{MAin RESUlts}

We now state the two main theorems of the paper. The proofs of Theorems 3.1 and 3.2 are given at the end of Section 7

Theorem 3.1. Let $A$ and $A^{\prime}$ be nondegenerate matrices with entries from $\{0,1\}$ satisfying Condition (II). For every isomorphism $(\kappa, \rho)$ from $\mathrm{FK}_{\text {red }}\left(\mathcal{O}_{A}\right)$ to $\mathrm{FK}_{\text {red }}\left(\mathcal{O}_{A^{\prime}}\right)$ there exists $a^{*}$-isomorphism $\Psi$ from $\mathcal{O}_{A} \otimes \mathbb{K}$ to $\mathcal{O}_{A^{\prime}} \otimes \mathbb{K}$ such that $\mathrm{FK}_{\text {red }}^{s}(\Psi)=(\kappa, \rho)$.

Theorem 3.2. Let $A$ and $A^{\prime}$ be nondegenerate matrices with entries from $\{0,1\}$ satisfying Condition (II). For every isomorphism $(\kappa, \rho)$ from $\mathrm{FK}_{\mathrm{red}}\left(\mathcal{O}_{A}\right)$ to $\mathrm{FK}_{\text {red }}\left(\mathcal{O}_{A^{\prime}}\right)$ that preserves the class of the unit, there exists $a^{*}$-isomorphism $\Psi$ from $\mathcal{O}_{A}$ to $\mathcal{O}_{A^{\prime}}$ such that $\mathrm{FK}_{\text {red }}(\Psi)=(\kappa, \rho)$.

We immediately get the following corollary, where the first half of course is already proved earlier in Res06.

Corollary 3.3. Let $A$ and $A^{\prime}$ be nondegenerate matrices with entries from $\{0,1\}$ satisfying Condition (II).

Then $\mathcal{O}_{A} \otimes \mathbb{K} \cong \mathcal{O}_{A^{\prime}} \otimes \mathbb{K}$ if and only if there exists an isomorphism from $\mathrm{FK}_{\text {red }}\left(\mathcal{O}_{A}\right)$ to $\mathrm{FK}_{\text {red }}\left(\mathcal{O}_{A^{\prime}}\right)$, and, moreover, $\mathcal{O}_{A} \cong \mathcal{O}_{A^{\prime}}$ if and only if there exists an isomorphism from $\mathrm{FK}_{\text {red }}\left(\mathcal{O}_{A}\right)$ to $\mathrm{FK}_{\text {red }}\left(\mathcal{O}_{A^{\prime}}\right)$ that preserves the class of the unit. 
3.1. Proofs of Theorems 3.1 and 3.2. The proofs of Theorem 3.1 and Theorem 3.2 will be carried out in the remaining sections of the paper. The idea of the proof of Theorem 3.1 is relatively simple. The isomorphism $(\kappa, \rho)$ is induced by a $\mathrm{GL}_{\mathcal{P}}$-equivalence between $A-I$ and $A^{\prime}-I$. By doing Cuntz-splices on $A^{\prime}$ (see Section (7), we can change this $\mathrm{GL}_{\mathcal{P}}$-equivalence into an $\mathrm{SL}_{\mathcal{P}}$-equivalence, and by the results of Boyle in Boy02 such an $\mathrm{SL}_{\mathcal{P}}$-equivalence can be decomposed into a composition of $\mathrm{SL}_{\mathcal{P}}$-equivalences given by elementary matrices. Finally, for each $\mathrm{SL}_{\mathcal{P}}$-equivalence given by an elementary matrix we can construct a stable isomorphism that induces the same map on the filtered $K$-theory as the $\mathrm{SL}_{\mathcal{P}}$-equivalence (see Section 6). Since $G_{\mathcal{P}}$-equivalences might take us out of the class of $\{0,1\}$ matrices, we work in the rest of the paper with graph $C^{*}$-algebras rather than Cuntz-Krieger algebras. Also for the benefit of later applications, we prove some results in slightly greater generality than we need - allowing for singular vertices sometimes. Theorem 3.2 will follow from Theorem 3.1 and [ERR13c, Theorem 3.3].

3.2. Definitions and notation. We adopt verbatim the definitions and the notation from [ERRS16b] - in particular Sections 2, 3 and 4. Since all notation needed is defined and explained in ERRS16b, we will only recall some of it here, but refer the reader to that paper for the rest.

Definition 3.4. For a countable graph $E=\left(E^{0}, E^{1}, r, s\right)$ (i.e., $E^{0}$ and $E^{1}$ are countable sets), we define its adjacency matrix $\mathrm{A}_{E}$ as an $E^{0} \times E^{0}$ matrix with the $(u, v)$ th entry being

$$
\left|\left\{e \in E^{1} \mid s(e)=u, r(e)=v\right\}\right| .
$$

Since $E$ is countable, $A_{E}$ will be a finite matrix or a countably infinite matrix, and it will have entries from $\mathbb{N}_{0} \sqcup\{\infty\}$.

It will be convenient for us to alter the adjacency matrix of a graph in two very specific ways, removing singular rows and subtracting the identity, so we introduce notation for this.

Notation 3.5. Let $E$ be a graph and $A_{E}$ its adjacency matrix. The matrix $A_{E}^{\bullet}$ will denote the matrix obtained from $A_{E}$ by removing all rows corresponding to singular vertices of $E$. We define $\mathrm{B}_{E}:=\mathrm{A}_{E}-I$ and let $\mathrm{B}_{E}^{\bullet}$ be $\mathrm{B}_{E}$ with the rows corresponding to singular vertices of $E$ removed. If $E$ is a finite graph with no sinks, then $\mathrm{B}_{E}^{\bullet}=\mathrm{B}_{E}$.

We will predominantly be working with graphs $E$ such that

$$
\begin{aligned}
& \mathrm{B}_{E}^{\bullet} \in \mathfrak{M}_{\mathcal{P}}(\mathbf{m} \times \mathbf{n}, \mathbb{Z}), \quad \mathrm{B}_{E} \in \mathfrak{M}_{\mathcal{P}}^{\circ}(\mathbf{m} \times \mathbf{n}, \mathbb{Z}), \\
& \mathrm{B}_{E} \in \mathfrak{M}_{\mathcal{P}}^{\circ \circ}(\mathbf{m} \times \mathbf{n}, \mathbb{Z}), \quad \text { or } \quad \mathrm{B}_{E} \in \mathfrak{M}_{\mathcal{P}}^{\circ \circ}(\mathbf{m} \times \mathbf{n}, \mathbb{Z})
\end{aligned}
$$

(see [ERRS16b, Definition 4.4 and Definition 4.15]). For the benefit of the reader we recall from [ERRS16b] the definitions of when $\mathrm{B}_{E}^{\bullet} \in \mathfrak{M}_{\mathcal{P}}(\mathbf{m} \times \mathbf{n}, \mathbb{Z}), \mathrm{B}_{E} \in$ $\mathfrak{M}_{\mathcal{P}}^{\circ}(\mathbf{m} \times \mathbf{n}, \mathbb{Z}), \mathrm{B}_{E} \in \mathfrak{M}_{\mathcal{P}}^{\circ \circ}(\mathbf{m} \times \mathbf{n}, \mathbb{Z})$, and $\mathrm{B}_{E} \in \mathfrak{M}_{\mathcal{P}}^{\circ \circ \circ}(\mathbf{m} \times \mathbf{n}, \mathbb{Z})$, as well as the definitions of $\mathfrak{M}(\mathbf{m} \times \mathbf{n}, \mathbb{Z}), \mathfrak{M}_{\mathcal{P}}(\mathbf{m} \times \mathbf{n}, \mathbb{Z}), \mathfrak{M}_{\mathcal{P}}(\mathbf{n}, \mathbb{Z}), \mathrm{GL}_{\mathcal{P}}(\mathbf{n}, \mathbb{Z})$, and $\operatorname{SL}_{\mathcal{P}}(\mathbf{n}, \mathbb{Z})$ below.

Notation 3.6. For $m, n \in \mathbb{N}_{0}$, let $\mathfrak{M}(m \times n, \mathbb{Z})$ denote the set of group homomorphisms from $\mathbb{Z}^{n}$ to $\mathbb{Z}^{m}$. When $m, n \geq 1$, we can equivalently view this as the $m \times n$ matrices over $\mathbb{Z}$. For $m=0$ or $n=0$, we similarly consider a group homomorphism from $\mathbb{Z}^{n}$ to $\mathbb{Z}^{m}$ as an empty matrix with zero rows or columns. Notice that composition of group homomorphisms corresponds to matrix multiplication. 
Suppose $N \in \mathbb{N}$ and let $\mathbf{m}=\left(m_{i}\right)_{i=1}^{N}, \mathbf{n}=\left(n_{i}\right)_{i=1}^{N} \in \mathbb{N}_{0}^{N}$. We let $\mathfrak{M}(\mathbf{m} \times \mathbf{n}, \mathbb{Z})$ denote the set of group homomorphisms from $\mathbb{Z}^{n_{1}} \oplus \cdots \oplus \mathbb{Z}^{n_{N}}$ to $\mathbb{Z}^{m_{1}} \oplus \cdots \oplus \mathbb{Z}^{m_{N}}$, and for such a homomorphism $B$, we let $B\{i, j\} \in \mathfrak{M}\left(m_{i} \times n_{j}, \mathbb{Z}\right)$ denote the component of $B$ from the $j$ th direct summand to the $i$ th direct summand. We also use the notation $B\{i\}$ for $B\{i, i\}$.

Let $\preceq$ be a partial order of the set $\{1,2, \ldots, N\}$ such that $i \preceq j \Longrightarrow i \leq j$. We use $\mathcal{P}$ to denote $\{1,2, \ldots, N\}$ viewed as a partially ordered set with the partial order $\preceq$. A morphism $B \in \mathfrak{M}(\mathbf{m} \times \mathbf{n}, \mathbb{Z})$ is said to be in $\mathfrak{M}_{\mathcal{P}}(\mathbf{m} \times \mathbf{n}, \mathbb{Z})$ if $B\{i, j\} \neq$ $0 \Longrightarrow i \preceq j$ for all $i, j \in \mathcal{P}$. We write $\mathfrak{M}_{\mathcal{P}}(\mathbf{n}, \mathbb{Z})$ for $\mathfrak{M}_{\mathcal{P}}(\mathbf{n} \times \mathbf{n}, \mathbb{Z})$ and denote by $\operatorname{GL}_{\mathcal{P}}(\mathbf{n}, \mathbb{Z})$ the automorphisms in $\mathfrak{M}_{\mathcal{P}}(\mathbf{n}, \mathbb{Z})$. Then $U \in \mathfrak{M}_{\mathcal{P}}(\mathbf{n}, \mathbb{Z})$ belongs to $\operatorname{GL}_{\mathcal{P}}(\mathbf{n}, \mathbb{Z})$ if and only if the determinant of the matrix defined by $U\{i\}$ is \pm 1 whenever $n_{i} \neq 0$. We denote by $\operatorname{SL}_{\mathcal{P}}(\mathbf{n}, \mathbb{Z})$ the automorphisms $U \in \mathrm{GL}_{\mathcal{P}}(\mathbf{n}, \mathbb{Z})$ for which the determinant of the matrix defined by $U\{i\}$ is 1 whenever $n_{i} \neq 0$.

Suppose $E=\left(E^{0}, E^{1}, r, s\right)$ is a countable graph with finitely many vertices. A nonempty subset $S$ of $E^{0}$ is strongly connected if for all $v, w \in E^{0}$ there is a nonempty path from $v$ to $w$. A strongly connected subset of $E^{0}$ is a strongly connected component if it is not a proper subset of another strongly connected subset. We let $\Gamma_{E}$ denote the set of all strongly connected components together with all singletons consisting of singular vertices that are not the base point of a cycle. The sets in $\Gamma_{E}$ are then mutually disjoint. A strongly connected component is called a cyclic component if all its vertices receive exactly one edge with source in the component. We define a relation $\geq$ on $\Gamma_{E}$ by letting $\gamma_{1} \geq \gamma_{2}$ if there is a path from a vertex in $\gamma_{1}$ to a vertex in $\gamma_{2}$. A vertex $v \in E^{0}$ is called a transition state if it does not belong to $\bigcup \Gamma_{E}$. A vertex is a transition state if and only if it is regular and is not the base of a cycle. For a subset $S$ of $E^{0}$, we denote by $H(S)$ the hereditary subset generated by $S$, and by $\bar{S}$ the saturation of $S$ (see [ERRS16b, Definition 3.1]).

Let $f_{E}$ be a map from $E^{0}$ to $\mathcal{P}$ such that if there is a path from $v$ to $w$, then $f_{E}(v) \preceq f_{E}(w)$. We say that $\mathrm{B}_{E}^{\bullet}$ is an element of $\mathfrak{M}_{\mathcal{P}}(\mathbf{m} \times \mathbf{n}, \mathbb{Z})$ and write $\mathrm{B}_{E}^{\bullet} \in$ $\mathfrak{M}_{\mathcal{P}}(\mathbf{m} \times \mathbf{n}, \mathbb{Z})$ if for all $i \in \mathcal{P}$, we have that $n_{i}$ is the number of vertices in $f_{E}^{-1}(\{i\})$, and $m_{i}$ is the number of vertices in $f_{E}^{-1}(\{i\})$ that are regular in $E$. We say that $\mathrm{B}_{E}$ is an element of $\mathfrak{M}_{\mathcal{P}}^{\circ}(\mathbf{m} \times \mathbf{n}, \mathbb{Z})$ and write $\mathrm{B}_{E} \in \mathfrak{M}_{\mathcal{P}}^{\circ}(\mathbf{m} \times \mathbf{n}, \mathbb{Z})$ if in addition the following conditions are satisfied: There is for each $i \in \mathcal{P}$ a unique $\gamma_{i} \in \Gamma_{E}$ such that $f_{E}\left(\gamma_{i}\right)=\{i\}, \gamma_{i} \geq \gamma_{j} \Longleftrightarrow i \preceq j$ for $i, j \in \mathcal{P}$, every infinite emitter emits infinitely many edges to any vertex it emits an edge to, every transition state has exactly one edge going out, and

$$
\overline{H\left(\gamma_{i}\right)} \backslash \overline{H\left(\gamma_{i}\right) \backslash \gamma_{i}}=f_{E}^{-1}(\{i\}) \quad \text { for all } i \in \mathcal{P} .
$$

We say that $\mathrm{B}_{E}$ is an element of $\mathfrak{M}_{\mathcal{P}}^{\circ}(\mathbf{m} \times \mathbf{n}, \mathbb{Z})$ and write $\mathrm{B}_{E} \in \mathfrak{M}_{\mathcal{P}}^{\circ \circ}(\mathbf{m} \times \mathbf{n}, \mathbb{Z})$ if $\mathrm{B}_{E} \in \mathfrak{M}_{\mathcal{P}}^{\circ}(\mathbf{m} \times \mathbf{n}, \mathbb{Z})$ and $E$ does not have any transition states, and we say that $\mathrm{B}_{E}$ is an element of $\mathfrak{M}_{\mathcal{P}}^{\circ \circ \circ}(\mathbf{m} \times \mathbf{n}, \mathbb{Z})$ and write $\mathrm{B}_{E} \in \mathfrak{M}_{\mathcal{P}}^{\circ \circ}(\mathbf{m} \times \mathbf{n}, \mathbb{Z})$ if $\mathrm{B}_{E} \in \mathfrak{M}_{\mathcal{P}}^{\circ \circ}(\mathbf{m} \times \mathbf{n}, \mathbb{Z})$ and every cyclic component $\gamma \in \Gamma_{E}$ consists of only one vertex.

When $\mathrm{B}_{E}^{\bullet} \in \mathfrak{M}_{\mathcal{P}}(\mathbf{m} \times \mathbf{n}, \mathbb{Z})$, then we will identify $\mathrm{B}_{E}^{\bullet}$ with the corresponding element of $\mathfrak{M}_{\mathcal{P}}(\mathbf{m} \times \mathbf{n}, \mathbb{Z})$ and $B_{E}$ with the corresponding element of $\mathfrak{M}_{\mathcal{P}}(\mathbf{n}, \mathbb{Z})$. Notice that these elements of $\mathfrak{M}_{\mathcal{P}}(\mathbf{m} \times \mathbf{n}, \mathbb{Z})$ and $\mathfrak{M}_{\mathcal{P}}(\mathbf{n}, \mathbb{Z})$, as well as the definitions of when $\mathrm{B}_{E}^{\bullet} \in \mathfrak{M}_{\mathcal{P}}(\mathbf{m} \times \mathbf{n}, \mathbb{Z}), \mathrm{B}_{E} \in \mathfrak{M}_{\mathcal{P}}^{\circ}(\mathbf{m} \times \mathbf{n}, \mathbb{Z}), \mathrm{B}_{E} \in \mathfrak{M}_{\mathcal{P}}^{\circ}(\mathbf{m} \times \mathbf{n}, \mathbb{Z})$, and $\mathrm{B}_{E} \in \mathfrak{M}_{\mathcal{P}}^{\circ \circ \circ}(\mathbf{m} \times \mathbf{n}, \mathbb{Z})$, depend on $f_{E}$ and the partial order $\preceq$. In order not to make 
the statement and proofs of the results too complicated, we do not specify how $f_{E}$ is chosen.

Notation 3.7. For an arbitrary unital graph $C^{*}$-algebra $C^{*}(E)$, we will consider a slightly different definition of reduced filtered $K$-theory. We refer the reader to ERRS16b, Definition 3.10, Definition 3.21, and Section 4.3], for the definition of $\mathrm{FK}_{\mathcal{R}}\left(X ; C^{*}(E)\right)$ and $\mathrm{FK}_{\mathcal{R}}\left(\mathcal{P} ; C^{*}(E)\right)$, where $X$ is a finite $T_{0}$-space and $\mathcal{P}$ is a partially ordered set such that $\mathrm{B}_{E} \in \mathfrak{M}_{\mathcal{P}}^{\circ}(\mathbf{m} \times \mathbf{n}, \mathbb{Z})$. When $E$ is a finite graph with no sinks and no sources that satisfies Condition $(\mathrm{K})$ (i.e., $C^{*}(E)$ is isomorphic to a purely infinite Cuntz-Krieger algebra) and $\mathrm{B}_{E} \in \mathfrak{M}_{\mathcal{P}}^{\circ}(\mathbf{m} \times \mathbf{n}, \mathbb{Z})$, then the definitions of $\mathrm{FK}_{\text {red }}\left(C^{*}(E)\right), \mathrm{FK}_{\mathcal{R}}\left(\operatorname{Prim} C^{*}(E) ; C^{*}(E)\right)$, and $\mathrm{FK}_{\mathcal{R}}\left(\mathcal{P} ; C^{*}(E)\right)$ are equivalent.

Remark 3.8. Let $E$ and $F$ be graphs such that $\mathrm{B}_{E}^{\bullet}$ and $\mathrm{B}_{F}^{\bullet}$ are elements in $\mathfrak{M}_{\mathcal{P}}(\mathbf{m} \times \mathbf{n}, \mathbb{Z})$. Let $(U, V)$ be a $\mathrm{GL}_{\mathcal{P}}(\mathbf{n}, \mathbb{Z})$-equivalence or an $\mathrm{SL}_{\mathcal{P}}(\mathbf{n}, \mathbb{Z})$-equivalence (see [ERRS16b, Definition 4.6]) from $\mathrm{B}_{E}^{\bullet}$ to $\mathrm{B}_{F}^{\bullet}$. As explained in ERRS16b, Section 4], in the induced isomorphisms on the reduced filtered $K$-theory, $\operatorname{FK}_{\mathcal{R}}(U, V)$ : $\mathrm{FK}_{\mathcal{R}}\left(\mathcal{P}, C^{*}(E)\right) \rightarrow \mathrm{FK}_{\mathcal{R}}\left(\mathcal{P}, C^{*}(F)\right), V^{\top}$ induces the map on $K_{0}$ and $\left(U^{\top}\right)^{-1}$ induces the map on $K_{1}$. Also, we let $\operatorname{FK}_{\mathcal{R}}^{s}(U, V)$ denote the canonically induced isomorphism from $\mathrm{FK}_{\mathcal{R}}\left(\mathcal{P}, C^{*}(E) \otimes \mathbb{K}\right)$ to $\mathrm{FK}_{\mathcal{R}}\left(\mathcal{P}, C^{*}(F) \otimes \mathbb{K}\right)$.

3.3. Strategy of proof and structure of the paper. The outline of the proof of Theorem 3.1 is as follows. We emulate the previous proofs that go from filtered $K$ theory data to stable isomorphism or flow equivalence, as in BH03, Boy02, Res06. A key component of those proofs is manipulation of the matrix $\mathrm{B}_{E}$, in particular that we can perform certain basic row and column operations without changing the stable isomorphism class or the flow equivalence class, depending on the context ( $c f$. ERRS16a, Propositions 3.2 and 3.5]). In Section 6. we show how to describe the isomorphisms these matrix operations induce on the (stabilized) graph $C^{*}$ algebras and their reduced filtered $K$-theory. In ERRS16c, it was proved that Cuntz splicing a graph gives a graph whose $C^{*}$-algebra is stably isomorphic to the $C^{*}$-algebra of the original graph (generalizing a result in Res06]). As we are proving a strong classification result, we will need to know more about what this stable isomorphism induces on the reduced filtered $K$-theory. We deal with this in Section 7 . We will also need to increase the size of our matrices using edge expansion and we keep track of the induced isomorphism on reduced filtered $K$ theory. This is done in Section 5 . Since the reduced filtered $K$-theory of $C^{*}(E)$ contains more groups than the $K$-web of $\mathrm{B}_{E}$, we need to strengthen some of the results of $\mathrm{BH} 03$. This is done in Section 4. Using these results, our proof of Theorem 3.1 goes through six steps.

Step 1. First, the homeomorphism $\rho$ from $\operatorname{Prim}\left(\mathcal{O}_{A}\right)$ to $\operatorname{Prim}\left(\mathcal{O}_{A^{\prime}}\right)$ allows us to view $\mathcal{O}_{A^{\prime}}$ as a $C^{*}$-algebra over $\operatorname{Prim}\left(\mathcal{O}_{A}\right)$. Thus, the $*$-isomorphism $\kappa: \mathcal{O}_{A} \rightarrow \mathcal{O}_{A^{\prime}}$ induces an isomorphism from $\operatorname{FK}_{\mathcal{R}}\left(\operatorname{Prim}\left(\mathcal{O}_{A}\right), \mathcal{O}_{A}\right)$ to $\operatorname{FK}_{\mathcal{R}}\left(\operatorname{Prim}\left(\mathcal{O}_{A}\right), \mathcal{O}_{A^{\prime}}\right)$.

Step 2. Second, we note that we might as well show the theorem for the graph $C^{*}$-algebras $C^{*}(E)$ and $C^{*}\left(E^{\prime}\right)$ instead of the Cuntz-Krieger algebras $\mathcal{O}_{A}$ and $\mathcal{O}_{A^{\prime}}$, where $E$ and $E^{\prime}$ are finite graphs satisfying Condition $(\mathrm{K})$ with no sinks and no sources, since every Cuntz-Krieger algebra is isomorphic to such a graph $C^{*}$-algebra. The reason is that it is important to our proof techniques to be able to work with matrices that are not $\{0,1\}$-matrices, since we will do row and column operations as mentioned above. 
Step 3. Now, we reduce the problem by finding graphs $E_{1}$ and $E_{1}^{\prime}$ in a certain standard form such that we have equivariant isomorphisms from $C^{*}(E) \otimes \mathbb{K}$ to $C^{*}\left(E_{1}\right) \otimes \mathbb{K}$ and from $C^{*}\left(E^{\prime}\right) \otimes \mathbb{K}$ to $C^{*}\left(E_{1}^{\prime}\right) \otimes \mathbb{K}$. This standard form will ensure that the matrices $A_{E_{1}}$ and $A_{E_{1}^{\prime}}$ have the same size and block structure, and that they satisfy certain additional technical conditions. This will be done in Lemmas 6.3 and 6.4

Step 4. In Section 4 we show a result similar to BH03, Corollary 4.7]. This result says that an isomorphism $\operatorname{FK}_{\mathcal{R}}\left(\mathcal{P}, C^{*}\left(E_{1}\right)\right) \cong \mathrm{FK}_{\mathcal{R}}\left(\mathcal{P}, C^{*}\left(E_{1}^{\prime}\right)\right)$ is induced by a $\mathrm{GL}_{\mathcal{P}}$-equivalence from $\mathrm{B}_{E_{1}}$ to $\mathrm{B}_{E_{1}^{\prime}}$.

Step 5. We then use the results in Section 7 to find graphs $E_{2}, E_{2}^{\prime}$ in a certain standard form such that we have equivariant isomorphisms from $C^{*}\left(E_{1}\right) \otimes \mathbb{K}$ to $C^{*}\left(E_{2}\right) \otimes \mathbb{K}$ and from $C^{*}\left(E_{1}^{\prime}\right) \otimes \mathbb{K}$ to $C^{*}\left(E_{2}^{\prime}\right) \otimes \mathbb{K}$ that induce isomorphisms on the reduced filtered $K$-theory that are induced by $\mathrm{GL}_{\mathcal{P}}$-equivalences such that the composed $\mathrm{GL}_{\mathcal{P}}$-equivalence from $\mathrm{B}_{E_{2}}$ to $\mathrm{B}_{E_{2}^{\prime}}$ is in fact an $\mathrm{SL}_{\mathcal{P}}$-equivalence.

Step 6. The result will now follow from Proposition 6.1 and Boy02, Theorem 4.4].

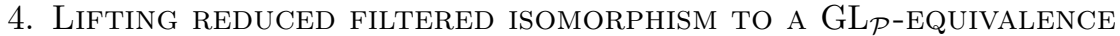

In $\mathrm{BH} 03$ there are many useful theorems concerning lifting $K$-web isomorphisms to $\mathrm{GL}_{\mathcal{P}}$-equivalences and $\mathrm{SL}_{\mathcal{P}}$-equivalences. Note that all $n_{i}$ 's are assumed to be nonzero in $\mathrm{BH} 03$. From [BH03, Theorems 4.4 and 4.5] we get the useful Corollary 4.7 in [BH03], that allows us to lift $K$-web isomorphisms to $\mathrm{GL}_{\mathcal{P}}$-equivalences provided that the gcd of each diagonal block is 1 . This corollary is easily generalized to also allow for diagonal blocks with $\operatorname{gcd} B\{i\}=\operatorname{gcd} B^{\prime}\{i\}=0$ (i.e., $B\{i\}$ and $B^{\prime}\{i\}$ are the zero matrices); this follows easily, since every automorphism of the cokernel of a (square) zero matrix is GL-allowable ( $c f$. [BH03]).

The reduced filtered $K$-theory includes more information in general than the $K$-webs (every $K_{1}$-group of every gauge simple subquotient; $c f$. the discussion in ERRS16b, Section 4.4]). Therefore, we will need to strengthen the result [BH03, Corollary 4.7] even more. First, we generalize [BH03, Theorem 4.4 (and Proposition 4.1)] as follows (we here only consider the case $\mathcal{R}=\mathbb{Z}$ ).

Theorem 4.1. Let $B$ be an $n \times n$ (square) matrix over $\mathbb{Z}$ with $n \in \mathbb{N}$, and let $\delta=\operatorname{gcd} B$. Let $\phi$ be an automorphism of $\operatorname{cok} B$, let $\psi$ be an automorphism of $\operatorname{ker} B$, and let $M$ be any $n \times n$ matrix over $\mathbb{Z}$ inducing $\phi$, i.e., $\phi([x])=[M x]$ for all $x \in \mathbb{Z}^{n}$.

Then $\operatorname{det}(M) \equiv \pm 1(\bmod \delta)$ if and only if there exist $n \times n$ invertible (GL) matrices $U$ and $V$ over $\mathbb{Z}$ such that $U B V=B$ and $U$ induces $\phi$ and $V^{-1}$ induces $\psi$.

Proof. The only thing that does not follow from [BH03. Theorem 4.4] is that we can choose the GL-equivalence $(U, V)$ such that it also induces the right automorphism on $\operatorname{ker} B$. For this, it is clear that we may assume that $B$ is its own Smith normal form (just like in the proof of [BH03, Theorem 4.4]). We use [BH03, Theorem 4.4] to get a GL-equivalence $\left(U, V^{\prime}\right): B \rightarrow B$ that induces $\phi$ on $\operatorname{cok} B$. The matrix $V^{\prime-1}$ induces an automorphism $\psi^{\prime}$ of $\operatorname{ker} B$. Now we will find a GL-equivalence $\left(I, V^{\prime \prime}\right): B \rightarrow B$ that induces $\psi \circ \psi^{\prime-1}$ on $\operatorname{ker} B-$ then $\left(U, V^{\prime} V^{\prime \prime}\right)$ is a GLequivalence that induces $\phi$ on $\operatorname{cok} B$ and $\psi$ on $\operatorname{ker} B$. Now, the automorphism $\psi \circ \psi^{\prime-1}$ on $\operatorname{ker} B$ uniquely determines what $V^{\prime \prime-1}$ should be on the lower right 
block matrix (where we write the matrices as $2 \times 2$ block matrices according to the nonzero, respectively zero, part of the diagonal of $B$ ). Let $V^{\prime \prime-1}$ be the block diagonal matrix that has this matrix as lower right block matrix and the identity as the upper left block matrix.

Now, we let

$$
\mathcal{P}_{\min }=\{i \in \mathcal{P} \mid j \preceq i \Rightarrow i=j\} .
$$

Using the above result, we get the following stronger version of [BH03, Theorem 4.5].

Theorem 4.2 (Strengthening of [BH03, Theorem 4.5]). Let $\mathbf{n}=\left(n_{i}\right)_{i \in \mathcal{P}}$ be a multi-index with $n_{i} \neq 0$, for all $i \in \mathcal{P}$. Suppose $B$ and $B^{\prime}$ are matrices in $\mathfrak{M}_{\mathcal{P}}(\mathbf{n}, \mathbb{Z})$ with corresponding diagonal blocks equal, and $\kappa: K(B) \rightarrow K\left(B^{\prime}\right)$ is a $K$-web isomorphism. Suppose that for each $i \in \mathcal{P}_{\min }$, we have an automorphism $\psi_{i}: \operatorname{ker} B\{i\} \rightarrow \operatorname{ker} B^{\prime}\{i\}$. Then there exist matrices $U, V \in \mathrm{GL}(\mathbf{n}, \mathbb{Z})$ such that we have a $\mathrm{GL}_{\mathcal{P}}$-equivalence $(U, V): B \rightarrow B^{\prime}$ satisfying $\kappa_{(U, V)}=\kappa$ if and only if each of the automorphisms $d_{i}: \operatorname{cok} B\{i\} \rightarrow \operatorname{cok} B^{\prime}\{i\}$ defined by $\kappa$ are GL-allowable moreover, the $\mathrm{GL}_{\mathcal{P}}$-equivalence can always be chosen such that $V^{-1}\{i\}$ induces $\psi_{i}$ for each $i \in \mathcal{P}_{\min }$.

Proof. The only thing that does not follow from [BH03, Theorem 4.5], is that we can choose the $\mathrm{GL}_{\mathcal{P}}$-equivalence $(U, V)$ such that it also induces the right automorphisms on $\operatorname{ker} B\{i\}, i \in \mathcal{P}_{\min }$. We choose a $\mathrm{GL}_{\mathcal{P}}$-equivalence $\left(U, V^{\prime}\right)$ according to [BH03, Theorem 4.5], so that it induces the given $K$-web isomorphism. For each $i \in \mathcal{P}_{\text {min }}$, this gives an automorphism $\psi_{i}^{\prime}$ of $\operatorname{ker} B\{i\}$. Now choose GL-equivalences $\left(I, V_{i}^{\prime \prime}\right)$ of $B\{i\}$ according to the proof of Theorem 4.1 so that $V_{i}^{\prime \prime-1}$ induces $\psi_{i} \circ \psi_{i}^{\prime-1}$ for each $i \in \mathcal{P}_{\min }$. Let $V^{\prime \prime}$ be the block matrix that is the identity matrix everywhere except that $V^{\prime \prime}\{i\}=V_{i}^{\prime \prime}$ for every $i \in \mathcal{P}_{\min }$. It is straightforward to verify that $\left(I, V^{\prime \prime}\right)$ is a $\mathrm{GL}_{\mathcal{P}}$-equivalence from $B^{\prime}$ to $B^{\prime}$, and that $\left(U, V^{\prime} V^{\prime \prime}\right)$ induces $\kappa$ and the desired automorphisms on the minimal components.

Together with Theorem 4.1 (using [BH03, Proposition 4.1(1)] and the discussion right after), this gives us the following stronger version of [BH03, Corollary 4.7].

Corollary 4.3 (Strengthening of [BH03, Corollary 4.7]). Let $\mathbf{n}=\left(n_{i}\right)_{i \in \mathcal{P}}$ be a multi-index with $n_{i} \neq 0$, for all $i \in \mathcal{P}$. Suppose $B$ and $B^{\prime}$ are matrices in $\mathfrak{M}_{\mathcal{P}}(\mathbf{n}, \mathbb{Z})$ such that $\operatorname{gcd} B\{i\}=\operatorname{gcd} B^{\prime}\{i\} \in\{0,1\}$ for all $i \in \mathcal{P}$. Then for any $K$-web isomorphism $\kappa$ from $K(B)$ to $K\left(B^{\prime}\right)$ and any family of isomorphisms $\psi_{i}: \operatorname{ker} B\{i\} \rightarrow \operatorname{ker} B^{\prime}\{i\}, i \in \mathcal{P}_{\min }$, there exist matrices $U, V \in \operatorname{GL}(\mathbf{n}, \mathbb{Z})$ such that we have a $\mathrm{GL}_{\mathcal{P}}$-equivalence $(U, V): B \rightarrow B^{\prime}$ satisfying $\kappa_{(U, V)}=\kappa$ and $V^{-1}\{i\}$ induces $\psi_{i}$ for each $i \in \mathcal{P}_{\min }$.

This allows us to lift reduced filtered $K$-theory isomorphisms to $\mathrm{GL}_{\mathcal{P}}$-equivalences, given that the diagonal blocks satisfy the relevant conditions about ged (cf. [ERRS16b, Section 4.4]).

\section{EDGE EXPANSION}

In this section we will look at edge expansion, what stable isomorphism between the resulting graph $C^{*}$-algebras it induces, and describe the induced map on the reduced filtered $K$-theory in terms of an $\mathrm{SL}_{\mathcal{P}}$-equivalence. We need these results to be able to enlarge our matrices while still keeping track of the induced maps on $K$-theory - the results are used in the proof of Lemma 6.4. 
The results in CET12 are important here, and we will recall the notation and results when needed.

Definition 5.1. Let $E=\left(E^{0}, E^{1}, r_{E}, s_{E}\right)$ be a graph and let $e_{0} \in E^{1}$. Set $v_{0}=$ $s_{E}\left(e_{0}\right)$. Let $F$ be the simple expansion graph at $e_{0}$ defined by

$$
F^{0}=E^{0} \sqcup\left\{\tilde{v}_{0}\right\} \quad \text { and } \quad F^{1}=\left(E^{1} \backslash\left\{e_{0}\right\}\right) \sqcup\left\{f_{1}, f_{2}\right\}
$$

where $\left.s_{F}\right|_{E^{1} \backslash\left\{e_{0}\right\}}=\left.s_{E}\right|_{E^{1} \backslash\left\{e_{0}\right\}},\left.r_{F}\right|_{E^{1} \backslash\left\{e_{0}\right\}}=\left.r_{E}\right|_{E^{1} \backslash\left\{e_{0}\right\}}, s_{F}\left(f_{1}\right)=v_{0}, r_{F}\left(f_{1}\right)=$ $\tilde{v}_{0}=s_{F}\left(f_{2}\right)$, and $r_{F}\left(f_{2}\right)=r_{E}\left(e_{0}\right)$.

For a group $G$ and a set $I$, we let $G^{I}$ denote the direct product over $I$ and let $G^{(I)}$ denote the direct sum over $I$.

Let $E$ be a graph. By [CET12, Proposition 3.8], there exists an isomorphism

$$
\chi_{0}^{E}: \operatorname{cok}\left(\left(\mathrm{B}_{E}^{\bullet}\right)^{\top}\right) \rightarrow K_{0}\left(C^{*}(E)\right)
$$

given by $\chi_{0}^{E}\left(\bar{e}_{v}\right)=\left[p_{v}\right]_{0}$, where $e_{v}$ is the element in $\mathbb{Z}^{\left(E^{0}\right)}$ that is 1 at the $v$ th coordinate and zero in all other coordinates and $\bar{e}_{v}$ is the image of $e_{v}$ in cok $\left(\left(\mathrm{B}_{E}^{\bullet}\right)^{\top}\right)$. The preimage of the positive cone of $K_{0}\left(C^{*}(E)\right)$ is generated by

$$
\left\{e_{v}\right\} \cup\left\{e_{v}-\sum_{e \in F} e_{r(e)} \mid v \in E_{\text {sing }}^{0}, F \subseteq s_{E}^{-1}(v), F \text { finite }\right\} .
$$

By [CET12, Proposition 3.8], there exists an isomorphism

$$
\chi_{1}^{E}: \operatorname{ker}\left(\left(\mathrm{B}_{E}^{\bullet}\right)^{\top}\right) \rightarrow K_{1}\left(C^{*}(E)\right)
$$

given by $\chi_{1}^{E}(\mathbf{x})=\left[U_{\mathbf{x}}\right]_{1}$. See the paragraph before CET12, Fact 3.6] for the definition of $U_{\mathbf{x}}$. We will describe $\left(\chi_{1}^{E}\right)^{-1}$. To do this, we recall the proof of CET12, Proposition 3.8].

Let $\mathcal{T}(E)$ be the Toeplitz algebra of $E$, i.e., $\mathcal{T}(E)$ is the universal $C^{*}$-algebra generated by a set of mutually orthogonal projections $\left\{q_{v} \mid v \in E^{0}\right\}$ and a set $\left\{t_{e} \mid e \in E^{1}\right\}$ of partial isometries satisfying the relations

$$
\begin{aligned}
& \text { - } t_{e}^{*} t_{f}=0 \text { if } e, f \in E^{1} \text { and } e \neq f, \\
& \text { - } t_{e}^{*} t_{e}=q_{r(e)} \text { for all } e \in E^{1} \text {, and, } \\
& \text { - } t_{e} t_{e}^{*} \leq q_{s(e)} \text { for all } e \in E^{1} \text {. }
\end{aligned}
$$

Whenever we have a set of mutually orthogonal projections $\left\{q_{v} \mid v \in E^{0}\right\}$ and and a set of partial isometries $\left\{t_{e} \mid e \in E^{1}\right\}$ in a $C^{*}$-algebra satisfying the above relations, then we call these elements a Toeplitz-Cuntz-Krieger E-family. Note that there exists a canonical surjective ${ }^{*}$-homomorphism $\pi_{E}: \mathcal{T}(E) \rightarrow C^{*}(E)$ such that $\pi_{E}\left(q_{v}\right)=p_{v}$ and $\pi_{E}\left(t_{e}\right)=s_{e}$ for all $v \in E^{0}$ and $e \in E^{1}$, where $\left\{p_{v}, s_{e} \mid v \in E^{0}, e \in E^{1}\right\}$ is a Cuntz-Krieger $E$-family generating $C^{*}(E)$.

As explained in the proof of [CET12, Proposition 3.8], there exist isomorphisms $\kappa_{E}$ from $K_{0}\left(\operatorname{ker}\left(\pi_{E}\right)\right)$ to $\mathbb{Z}^{\left(E_{\text {reg }}^{0}\right)}$ and $\lambda_{E}$ from $K_{0}(\mathcal{T}(E))$ to $\mathbb{Z}^{\left(E^{0}\right)}$ such that the diagram

$$
\begin{gathered}
0 \longrightarrow K_{1}\left(C^{*}(E)\right) \stackrel{\partial_{1}^{E}}{\longrightarrow} K_{0}\left(\operatorname{ker}\left(\pi_{E}\right)\right) \stackrel{\left(\iota_{E}\right)_{*}}{\longrightarrow} K_{0}(\mathcal{T}(E)) \stackrel{\left(\pi_{E}\right)_{*}}{\longrightarrow} K_{0}\left(C^{*}(E)\right) \longrightarrow 0 \\
\mathbb{Z}^{\left(E_{\mathrm{reg}}^{0}\right)} \stackrel{\downarrow_{E}}{\longrightarrow} \underset{\left(\mathrm{B}_{E}^{*}\right)^{\top}}{\longrightarrow} \mathbb{Z}^{\left(E^{0}\right)}
\end{gathered}
$$

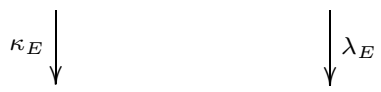


commutes, with the top row being the exact sequence in $K$-theory induced by

$$
0 \longrightarrow \operatorname{ker}\left(\pi_{E}\right) \stackrel{\iota_{E}}{\longrightarrow} \mathcal{T}(E) \stackrel{\pi_{E}}{\longrightarrow} C^{*}(E) \longrightarrow 0 .
$$

Moreover, $\lambda_{E}\left(\left[q_{v}\right]_{0}\right)=e_{v}$ and

$$
\kappa_{E}\left(\left[q_{w}-\sum_{s(e)=w} t_{e} t_{e}^{*}\right]\right)=e_{w}
$$

for all $v \in E^{0}$ and $w \in E_{\mathrm{reg}}^{0}$. By the proof of [CET12, Proposition 3.8], $\left(\chi_{1}^{E}\right)^{-1}=$ $\kappa_{E} \circ \partial_{1}^{E}$.

Suppose $E$ is a graph such that $\mathrm{B}_{E} \in \mathfrak{M}_{\mathcal{P}}^{\circ}(\mathbf{m} \times \mathbf{n}, \mathbb{Z})$. For a saturated, hereditary subset $H$ of $E^{0}$, we let $\mathfrak{J}_{H}$ denote the ideal in $C^{*}(E)$ generated by the vertex projections $\left\{p_{v} \mid v \in H\right\}$. For saturated hereditary subsets $H_{0} \subseteq H \subseteq E^{0}$, we now show how to identify $K_{0}\left(\mathfrak{J}_{H} / \mathfrak{J}_{H_{0}}\right)$ and $K_{1}\left(\mathfrak{J}_{H} / \mathfrak{J}_{H_{0}}\right)$ with the cokernel and kernel of a certain submatrix of $\left(\mathrm{B}_{E}^{\bullet}\right)^{\mathrm{T}}$. If $B$ is a matrix whose rows and columns are indexed by a set $S$ and $S_{0}$ is a subset of $S$, then we let $B\left\langle S_{0}\right\rangle$ denote the principal submatrix of $B$ whose $(i, j)$ entry, for $i, j \in S_{0}$, is the $(i, j)$ entry of $B$.

Let $H$ be a saturated hereditary subset of $E^{0}$. Let $E_{H}$ be the subgraph of $E$ given by $E_{H}^{0}=H, E_{H}^{1}=s_{E}^{-1}(H)$, and the source and range maps of $E_{H}$ are the maps inherited from $E$. Note that each saturated hereditary subset of $E_{H}^{0}$ is of the form $H_{0} \subseteq H$, with $H_{0}$ a saturated hereditary subset of $E^{0}$. Let $H_{0}$ be a saturated hereditary subset of $E^{0}$ with $H_{0} \subseteq H$. Let $E_{H \backslash H_{0}}$ be the subgraph of $E$ given by $E_{H \backslash H_{0}}^{0}=H \backslash H_{0}$ and $E_{H \backslash H_{0}}^{1}=s_{E}^{-1}(H) \cap r_{E}^{-1}\left(H \backslash H_{0}\right)$, and the source and range maps of $E_{H \backslash H_{0}}$ is the restriction of $s_{E}$ and $r_{E}$, respectively. Note that $E_{H \backslash H_{0}}$ is also a subgraph of $E_{H}$. There is an injective ${ }^{*}$-homomorphism $\Phi_{H \backslash H_{0}}: C^{*}\left(E_{H \backslash H_{0}}\right) \rightarrow$

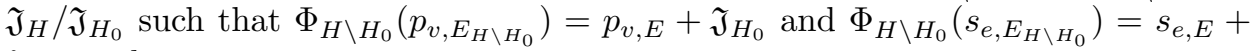
$\mathfrak{J}_{H_{0}}$, and

$$
\Phi_{H \backslash H_{0}}\left(C^{*}\left(E_{H \backslash H_{0}}\right)\right)=\bar{P}_{H \backslash H_{0}}\left(\mathfrak{J}_{H} / \mathfrak{J}_{H_{0}}\right) \bar{P}_{H \backslash H_{0}},
$$

where $\bar{P}_{H \backslash H_{0}}=\sum_{v \in H \backslash H_{0}}\left(p_{v, E}+\mathfrak{J}_{H_{0}}\right)$. Since $\bar{P}_{H \backslash H_{0}}\left(\mathfrak{J}_{H} / \mathfrak{J}_{H_{0}}\right) \bar{P}_{H \backslash H_{0}}$ is full in $\mathfrak{J}_{H} / \mathfrak{J}_{H_{0}}$, it follows from Bro77] (see also [RR07, Corollary 3.3]) that $K_{*}\left(\Phi_{H \backslash H_{0}}\right.$ ) is an isomorphism. Note that $\mathrm{A}_{E_{H \backslash H_{0}}}=\left(\mathrm{A}_{E}\right)\left\langle H \backslash H_{0}\right\rangle$ and $\mathrm{B}_{E_{H \backslash H_{0}}^{\bullet}}$ is the matrix obtained from $\mathrm{B}_{E}^{\bullet}$ by removing all rows and columns whose index does not belong to $H \backslash H_{0}$. Then

$$
\chi_{0}^{H \backslash H_{0}}:=K_{0}\left(\Phi_{H \backslash H_{0}}\right) \circ \chi_{0}^{E_{H \backslash H_{0}}}: \operatorname{cok}\left(\left(\mathrm{B}_{E_{H \backslash H_{0}}^{\bullet}}\right)^{\top}\right) \rightarrow K_{0}\left(\mathfrak{J}_{H} / \mathfrak{J}_{H_{0}}\right)
$$

and

$$
\chi_{1}^{H \backslash H_{0}}:=K_{1}\left(\Phi_{H \backslash H_{0}}\right) \circ \chi_{1}^{E_{H \backslash H_{0}}}: \operatorname{ker}\left(\left(\mathrm{B}_{E_{H \backslash H_{0}}}\right)^{\mathrm{T}}\right) \rightarrow K_{1}\left(\mathfrak{J}_{H} / \mathfrak{J}_{H_{0}}\right)
$$

are isomorphisms. See also BHRS02, Proposition 3.4].

Lemma 5.2. Let $E=\left(E^{0}, E^{1}, r_{E}, s_{E}\right)$ be a graph and let $e_{0} \in E^{1}$. Let $F$ be the simple expansion graph at $e_{0}$. Then there exists $a^{*}$-homomorphism $\Psi: \mathcal{T}(E) \rightarrow$ $\mathcal{T}(F)$ and an injective ${ }^{*}$-homomorphism $\bar{\Psi}: C^{*}(E) \rightarrow C^{*}(F)$ such that

(1) $\pi_{F} \circ \Psi=\bar{\Psi} \circ \pi_{E}$ (in particular, $\left.\Psi\right|_{\operatorname{ker}\left(\pi_{E}\right)}$ is a ${ }^{*}$-homomorphism from $\operatorname{ker}\left(\pi_{E}\right)$ to $\left.\operatorname{ker}\left(\pi_{F}\right)\right)$,

(2) $\bar{\Psi}\left(C^{*}(E)\right)=P C^{*}(F) P$, where $P=\sum_{v \in E^{0}} p_{v, F}$ is an element of the multiplier algebra of $C^{*}(F)$, 
(3) $\Psi\left(q_{v, E}\right)=q_{v, F}$ for all $v \in E^{0}$, and

(4) $\Psi\left(t_{e, E}\right)=t_{e, F}$ for all $e \in E^{1} \backslash\left\{e_{0}\right\}$,

where $\left\{q_{v, E}, t_{e, E} \mid v \in E^{0}, e \in E^{1}\right\}$ is a Toeplitz-Cuntz-Krieger E-family generating $\mathcal{T}(E)$ and $\left\{q_{v, F}, t_{e, F} \mid v \in F^{0}, e \in F^{1}\right\}$ is a Toeplitz-Cuntz-Krieger F-family generating $\mathcal{T}(F)$.

Proof. Define a Toeplitz-Cuntz-Krieger $E$-family in $\mathcal{T}(F)$ by setting $Q_{v}=q_{v, F}$ for all $v \in E^{0} \subseteq F^{0}$ and

$$
T_{e}= \begin{cases}t_{e, F} & \text { if } e \neq e_{0}, \\ t_{f_{1}, F} t_{f_{2}, F} & \text { if } e=e_{0},\end{cases}
$$

for $e \in E^{1}$. One checks that $\left\{Q_{v}, T_{e} \mid v \in E^{0}, e \in E^{1}\right\}$ is a Toeplitz-Cuntz-Krieger $E$-family in $\mathcal{T}(F)$. It follows that there exists a unique ${ }^{*}$-homomorphism $\Psi: \mathcal{T}(E) \rightarrow$ $\mathcal{T}(F)$ such that $\Psi\left(q_{v, E}\right)=Q_{v}$ and $\Psi\left(t_{e, E}\right)=T_{e}$.

Set $P_{v}=\pi_{F}\left(Q_{v}\right)$ and $S_{e}=\pi_{F}\left(T_{e}\right)$. It is easy to check that $\left\{P_{v}, S_{e} \mid v \in E^{0}\right.$, $\left.e \in E^{1}\right\}$ is a Cuntz-Krieger $E$-family in $C^{*}(F)$. It follows that there is a unique ${ }^{*}$-homomorphism $\bar{\Psi}: C^{*}(E) \rightarrow C^{*}(F)$ such that $\bar{\Psi}\left(p_{v, E}\right)=P_{v}$ and $\Psi\left(s_{e, E}\right)=S_{e}$. By [Szy02, Theorem 1.2], $\bar{\Psi}$ is injective. Since $P C^{*}(F) P$ is equal to the closed linear span of elements of the form $s_{\mu, F} s_{\nu, F}^{*}$ with $r_{F}(\mu)=r_{F}(\nu)$ and $s_{F}(\mu), s_{F}(\nu) \in E^{0}$ and since $s_{f_{1}, F} s_{f_{1}, F}^{*}=s_{f_{1} f_{2}, F} s_{f_{1} f_{2}, F}^{*}, \bar{\Psi}\left(C^{*}(E)\right)=P C^{*}(F) P$. Thus, (11) and (2) hold, and by construction (3) and (4) hold.

Remark 5.3. Suppose in Lemma 5.2 that $e_{0}$ is an edge on a cycle based at $v_{0}:=$ $s_{E}\left(e_{0}\right)$ and that $\mathrm{B}_{E} \in \mathfrak{M}_{\mathcal{P}}^{\circ}(\mathbf{m} \times \mathbf{n}, \mathbb{Z})$. Assume that $j \in \mathcal{P}$ corresponds to the component containing $v_{0}$. Then for the simple expansion graph $F$ at $e_{0}$, we have that $\mathrm{B}_{F} \in \mathfrak{M}_{\mathcal{P}}^{\circ}\left(\left(\mathbf{m}+\mathbf{e}_{j}\right) \times\left(\mathbf{n}+\mathbf{e}_{j}\right), \mathbb{Z}\right)$, where $\mathbf{e}_{j}$ is the multi-index that is 1 in the $j$ th coordinate and zero elsewhere. Moreover, there is a one-to-one correspondence between saturated hereditary subsets of $E^{0}$ and saturated hereditary subsets of $F^{0}$ given by

$$
H \mapsto \widetilde{H}= \begin{cases}H & \text { if } v_{0} \notin H, \\ H \cup\left\{\tilde{v}_{0}\right\} & \text { if } v_{0} \in H .\end{cases}
$$

Let $\bar{\Psi}_{E}: C^{*}(E) \rightarrow C^{*}(F)$ be the ${ }^{*}$-homomorphism given in Lemma 5.2 for the graph $E$. Since $\bar{\Psi}_{E}$ maps $\mathfrak{J}_{H}$ to $\mathfrak{J}_{\widetilde{H}}$ for all hereditary and saturated subsets $H \subseteq E^{0}$, $\bar{\Psi}_{E}$ induces a ${ }^{*}$-homomorphism, which we again denote by $\bar{\Psi}_{E}$, from $\mathfrak{J}_{H} / \mathfrak{J}_{H_{0}}$ to $\mathfrak{J}_{\widetilde{H}} / \mathfrak{J}_{\widetilde{H}_{0}}$.

Suppose $H$ is a saturated hereditary subset of $E^{0}$ containing $v_{0}$ and $H_{0}$ is a saturated hereditary subset of $E^{0}$ not containing $v_{0}$. Then $F_{\widetilde{H} \backslash \widetilde{H}_{0}}$ is the simple expansion of the graph $E_{H \backslash H_{0}}$ at $e_{0}$. Moreover, $\bar{\Psi}_{E} \circ \Phi_{H \backslash H_{0}}^{E}=\Phi_{\widetilde{H} \backslash \widetilde{H}_{0}}^{F} \circ \bar{\Psi}_{E_{H \backslash H_{0}}}$. Here $\Phi_{H \backslash H_{0}}^{E}$ is the embedding of $C^{*}\left(E_{H \backslash H_{0}}\right)$ into $\mathfrak{J}_{H} / \mathfrak{J}_{H_{0}}, \Phi_{\widetilde{H} \backslash \widetilde{H}_{0}}^{F}$ is the embedding of $C^{*}\left(F_{\widetilde{H} \backslash \widetilde{H}_{0}}\right)$ into $\mathfrak{J}_{\widetilde{H}} / \mathfrak{J}_{\widetilde{H}_{0}}$, and $\bar{\Psi}_{E_{H \backslash H_{0}}}$ is the ${ }^{*}$-homomorphism from $C^{*}\left(E_{H \backslash H_{0}}\right)$ to $C^{*}\left(F_{\widetilde{H} \backslash \widetilde{H}_{0}}\right)$ given in Lemma 5.2 for the graph $E_{H \backslash H_{0}}$.

We now describe how symbol expansion gives rise to an $\mathrm{SL}_{\mathcal{P}}$-equivalence and to a $\mathcal{P}$-equivariant stable isomorphism that induces the same map on the filtered $K$-theory as the $\mathrm{SL}_{\mathcal{P}}$-equivalence. This will be used in the proof of Lemma 6.4. 
Proposition 5.4. Let $E$ be a graph with finitely many vertices, and let $v_{0}$ be a vertex in $E^{0}$ that supports a cycle $\mu=\mu_{1} \cdots \mu_{n}$. Assume that $\mathrm{B}_{E} \in \mathfrak{M}_{\mathcal{P}}^{\circ}(\mathbf{m} \times \mathbf{n}, \mathbb{Z})$, and that $v_{0}$ belongs to the block $j \in \mathcal{P}$. Let $F$ be the graph in Definition 5.1 with $e_{0}=$ $\mu_{1}$ (i.e., the graph is obtained with a simple expansion of $e_{0}$ ). Then $E$ and $F$ are move equivalent, denoted by $E \sim_{M E} F$, as defined in [ERRS16b, Definition 2.19].

Moreover, $\mathrm{B}_{F} \in \mathfrak{M}_{\mathcal{P}}^{\circ}\left(\left(\mathbf{m}+\mathbf{e}_{j}\right) \times\left(\mathbf{n}+\mathbf{e}_{j}\right), \mathbb{Z}\right)$ and there exist $U \in \mathrm{SL}_{\mathcal{P}}\left(\mathbf{m}+\mathbf{e}_{j}, \mathbb{Z}\right)$ and $V \in \mathrm{SL}_{\mathcal{P}}\left(\mathbf{n}+\mathbf{e}_{j}, \mathbb{Z}\right)$ that are the identity matrices everywhere except for the $j$ th diagonal block, such that $(U, V)$ is an $\mathrm{SL}_{\mathcal{P}}$-equivalence from $-\iota_{\mathbf{e}_{j}}\left(-\mathrm{B}_{E}^{\bullet}\right)$ to $\mathrm{B}_{F}^{\bullet}$ where $\iota_{\mathbf{e}_{j}}$ is the embedding of $\mathfrak{M}_{\mathcal{P}}(\mathbf{m} \times \mathbf{n}, \mathbb{Z})$ into $\mathfrak{M}_{\mathcal{P}}\left(\left(\mathbf{m}+\mathbf{e}_{j}\right) \times\left(\mathbf{n}+\mathbf{e}_{j}\right), \mathbb{Z}\right)$ defined in [ERRS16b, Definition 4.7], and such that there exists a $\mathcal{P}$-equivariant isomorphism $\Phi$ from $C^{*}(E) \otimes \mathbb{K}$ to $C^{*}(F) \otimes \mathbb{K}$ satisfying $\mathrm{FK}_{\mathcal{R}}(\mathcal{P} ; \Phi)=\mathrm{FK}_{\mathcal{R}}^{s}(U, V)$.

Proof. We prove the case where $v_{0}$ is a regular vertex. The case $v_{0}$ is an infinite emitter is similar. We index the columns and rows of $-\iota_{\mathbf{e}_{j}}\left(-\mathrm{B}_{E}^{\bullet}\right)$ with $F^{0}$. The $j$ th component is indexed by $S_{0}=\left(H \backslash H_{0}\right) \sqcup\left\{\widetilde{v}_{0}\right\}$ for the saturated hereditary subsets $H=\overline{H\left(v_{0}\right)}$ and $H_{0}$ of $E^{0}$ where $H_{0}$ is the largest proper saturated hereditary subset of $H$ ( $c f$. [ERRS16b, Lemma 3.17(ii)]). Let $U$ be the identity matrix except for the $j$ th diagonal block and the $U\{j\}$ block is the basic elementary matrix that acts on the left by subtracting row $\tilde{v}_{0}$ from row $v_{0}$. Let $V$ be the identity matrix except for the $j$ th diagonal block and the $V\{j\}$ block is the basic elementary matrix that acts on the right by subtracting column $\tilde{v}_{0}$ from column $r\left(\mu_{1}\right)$. A computation shows that $(U, V)$ is an $\mathrm{SL}_{\mathcal{P}}$-equivalence from $-\iota_{\mathbf{e}_{j}}\left(-\mathrm{B}_{E}^{\bullet}\right)$ to $\mathrm{B}_{F}^{\bullet}$.

Clearly, $V^{\top}\left(e_{v}\right)=e_{v}$ for all $v \in E^{0}$. Moreover, for every $v \in F_{\text {reg }}^{0}$

$$
\left(U^{\top}\right)^{-1}\left(e_{v}\right)= \begin{cases}e_{v}, & \text { if } v \neq v_{0}, \\ e_{v_{0}}+e_{\widetilde{v}_{0}}, & \text { if } v=v_{0}\end{cases}
$$

Let $\bar{\Psi}: C^{*}(E) \rightarrow C^{*}(F)$ be the ${ }^{*}$-homomorphism given in Lemma [5.2. By Remark 5.3. we have that $\bar{\Psi}$ is a $\mathcal{P}$-equivariant homomorphism. We now show that $\bar{\Psi}$ induces $\mathrm{FK}_{\mathcal{R}}(U, V)$. Since $\bar{\Psi}\left(p_{v, E}\right)=p_{v, F}$ and $V^{\top}\left(e_{v}\right)=e_{v}$ for all $v \in E^{0}, \bar{\Psi}$ induces the desired maps on $K_{0}$. Let $S \subseteq F^{0}$ represent the $i$ th component with $i \neq j$. Since $v_{0} \notin S$, we have that $e_{0} \notin s^{-1}(S) \cap r^{-1}(S)$. Thus, $\bar{\Psi}\left(p_{v, E}\right)=p_{v, F}$ and $\bar{\Psi}\left(s_{e, E}\right)=s_{e, F}$ for all $v \in S$ and $e \in s^{-1}(S) \cap r^{-1}(S)$. By Remark 5.3. we have that $\bar{\Psi}$ induces the identity on the $K_{1}$-group of the $i$ th component.

We now show that $\bar{\Psi}$ induces $\mathrm{FK}_{\mathcal{R}}(U, V)$ on the $j$ th component. By Remark 5.3 , it is enough to show this for $E$ with $U=U\{j\}$ and $V=V\{j\}$. Let $\Psi: \mathcal{T}(E) \rightarrow$ $\mathcal{T}(F)$ be the ${ }^{*}$-homomorphism given in Lemma 5.2 , Let

$$
\mathbf{x}=\sum_{v \in E_{\mathrm{reg}}^{0}} x_{v} e_{v} \in \operatorname{ker}\left(\left(\mathrm{B}_{E}^{\bullet}\right)^{\top}\right)
$$

By the proof of [CET12, Proposition 3.8],

$$
\partial_{1}^{E}\left(\left[\mathrm{U}_{\mathbf{x}}\right]_{1}\right)=\sum_{x_{v} \neq 0} x_{v}\left[q_{v, E}-\sum_{s_{E}(e)=v} t_{e, E} t_{e, E}^{*}\right]_{0} .
$$


Therefore

$$
\begin{aligned}
\left(\partial_{1}^{F} \circ K_{1}(\bar{\Psi})\right)\left(\left[\mathrm{U}_{\mathbf{x}}\right]_{1}\right)= & \left(K_{0}(\Psi) \circ \partial_{1}^{E}\right)\left(\left[\mathrm{U}_{\mathbf{x}}\right]_{1}\right) \\
= & \sum_{x_{v} \neq 0} x_{v}\left[\Psi\left(q_{v, E}\right)-\sum_{s_{E}(e)=v} \Psi\left(t_{e, E}\right) \Psi\left(t_{e, E}\right)^{*}\right]_{0} \\
= & \sum_{\substack{x_{v} \neq 0 \\
v \neq v_{0}}} x_{v}\left[q_{v, F}-\sum_{s_{E}(e)=v} t_{e, F} t_{e, F}^{*}\right]_{0} \\
& +x_{v_{0}}\left[q_{v_{0}, F}-t_{f_{1}, F} t_{f_{2}, F} t_{f_{2}, F}^{*} t_{f_{1}, F}^{*}-\sum_{\substack{s_{E}(e)=v_{0} \\
e \neq e_{0}}} t_{e, F} t_{e, F}^{*}\right]_{0} .
\end{aligned}
$$

Note that in $K_{0}\left(\operatorname{ker}\left(\pi_{F}\right)\right)$,

$$
\begin{aligned}
& {\left[q_{v_{0}, F}-t_{f_{1}, F} t_{f_{2}, F} t_{f_{2}, F}^{*} t_{f_{1}, F}^{*}-\sum_{\substack{s_{E}(e)=v_{0} \\
e \neq e_{0}}} t_{e, F} t_{e, F}^{*}\right]^{2} } \\
= & {\left[q_{v_{0}, F}-t_{f_{1}, F} t_{f_{1}, F}^{*}-\sum_{\substack{s_{E}(e)=v_{0} \\
e \neq e_{0}}} t_{e, F} t_{e, F}^{*}\right]_{0}+\left[t_{f_{1}, F} t_{f_{1}, F}^{*}-t_{f_{1}, F} t_{f_{2}, F} t_{f_{2}, F}^{*} t_{f_{1}, F}^{*}\right]_{0} } \\
= & {\left[q_{v_{0}, F}-\sum_{s_{F}(e)=v_{0}} t_{e, F} t_{e, F}^{*}\right]_{0}+\left[t_{f_{1}, F}\left(q_{\tilde{v}_{0}, F}-t_{f_{2}, F} t_{f_{2}, F}^{*}\right) t_{f_{1}, F}^{*}\right]_{0} } \\
= & {\left[q_{v_{0}, F}-\sum_{s_{F}(e)=v_{0}} t_{e, F} t_{e, F}^{*}\right]_{0}+\left[q_{\tilde{v}_{0}, F}-t_{f_{2}, F} t_{f_{2}, F}^{*}\right]_{0} . }
\end{aligned}
$$

Hence,

$$
\kappa_{F} \circ\left(\partial_{1}^{F} \circ K_{1}(\bar{\Psi})\right)\left(\left[\mathrm{U}_{\mathbf{x}}\right]_{1}\right)=\sum_{\substack{x_{v} \neq 0 \\ v \neq v_{0}}} x_{v} e_{v}+x_{v_{0}} e_{v_{0}}+x_{v_{0}} e_{\tilde{v}_{0}}=\sum_{x_{v} \neq 0} x_{v} e_{v}+x_{v_{0}} e_{\tilde{v}_{0}} .
$$

Thus, $K_{1}(\bar{\Psi})$ is induced by $\left(U^{\top}\right)^{-1}$. We have just shown that $\operatorname{FK}_{\mathcal{R}}(\mathcal{P} ; \bar{\Psi})=$ $\mathrm{FK}_{\mathcal{R}}(U, V)$.

By Lemma 5.2, $\bar{\Psi}\left(C^{*}(E)\right)=P C^{*}(F) P$, where $P=\sum_{v \in E^{0}} p_{v, F}$. Note that $P C^{*}(F) P$ is full in $C^{*}(F)$, and by [Bro77, there exists a partial isometry $W$ in $\mathcal{M}\left(C^{*}(F) \otimes \mathbb{K}\right)$ such that $W^{*} W=P \otimes 1_{\mathbb{B}\left(\ell^{2}\right)}$ and $W W^{*}=1_{\mathcal{M}\left(C^{*}(F)\right)} \otimes 1_{\mathbb{B}\left(\ell^{2}\right)}$, where $\mathcal{M}\left(C^{*}(F) \otimes \mathbb{K}\right)$ and $\mathcal{M}\left(C^{*}(F)\right)$ are the multiplier algebras of $C^{*}(F) \otimes \mathbb{K}$ and $C^{*}(F)$, respectively. Since $\bar{\Psi}$ is a $\mathcal{P}$-equivariant homomorphism, we get from $\operatorname{Remark} 5.3$ that $\Phi=\operatorname{Ad}(W) \circ\left(\bar{\Psi} \otimes \mathrm{id}_{\mathbb{K}}\right): C^{*}(E) \otimes \mathbb{K} \rightarrow C^{*}(F) \otimes \mathbb{K}$ is a $\mathcal{P}$-equivariant isomorphism. It follows from [RR07] that $\mathrm{FK}_{\mathcal{R}}(\mathcal{P} ; \Phi)=\mathrm{FK}_{\mathcal{R}}\left(\mathcal{P} ; \bar{\Psi} \otimes \mathrm{id}_{\mathbb{K}}\right)$ and hence that $\operatorname{FK}_{\mathcal{R}}(\mathcal{P} ; \Phi)=\mathrm{FK}_{\mathcal{R}}^{s}(U, V)$. 


\section{Stable isomorphisms induced By Flow EQUivalences AND THEIR $K$-THEORY}

Following the work of Boyle in Boy02, we will break $\mathrm{SL}_{\mathcal{P}}$-equivalences into $\mathrm{SL}_{\mathcal{P}}$-equivalences induced by basic elementary matrices. It is well known that such $\mathrm{SL}_{\mathcal{P}}$-equivalences induced by basic elementary matrices induce stable isomorphisms. They also induce isomorphisms between the $K$-webs in a canonical way (and between the reduced filtered $K$-theories of the corresponding graph $C^{*}$-algebras).

In this section we will show that we can choose the stable isomorphisms above in such a way that the above operations commute.

Proposition 6.1. Let $E$ and $F$ be graphs such that $\mathrm{B}_{E}$ and $\mathrm{B}_{F}$ belong to $\mathfrak{M}_{\mathcal{P}}^{\circ \infty}(\mathbf{m} \times \mathbf{n}, \mathbb{Z})$. Let $u, v \in E^{0}$ with $u \neq v$ and $\mathrm{A}_{E}(u, v)>0$ and let $W \in \operatorname{SL}_{\mathcal{P}}(\mathbf{n}, \mathbb{Z})$ denote the positive basic elementary matrix with $W(u, v)=1$ and equal to the identity everywhere else.

Assume that $W \mathrm{~B}_{E}=\mathrm{B}_{F}$ and the vertex $v$ in $E$ is regular. Then $\left(W^{\bullet}, I\right)$ is an $\mathrm{SL}_{\mathcal{P}}$-equivalence from $\mathrm{B}_{E}^{\bullet}$ to $\mathrm{B}_{F}^{\bullet}$, where $W^{\bullet}$ is the matrix we get from $W$ by removing all columns and rows corresponding to singular vertices of $E$. Moreover, there exists a $\mathcal{P}$-equivariant isomorphism $\Phi$ from $C^{*}(E)$ to $C^{*}(F)$ such that $\mathrm{FK}_{\mathcal{R}}(\mathcal{P} ; \Phi)=$ $\mathrm{FK}_{\mathcal{R}}\left(W^{\bullet}, I\right)$.

Now assume instead that $\mathrm{B}_{E} W=\mathrm{B}_{F}$ and the vertex $u$ emits at least two edges. Then $(I, W)$ is an $\mathrm{SL}_{\mathcal{P}}$-equivalence from $\mathrm{B}_{E}^{\bullet}$ to $\mathrm{B}_{F}^{\bullet}$. Moreover, there exists a $\mathcal{P}$ equivariant isomorphism $\Phi$ from $C^{*}(E) \otimes \mathbb{K}$ to $C^{*}(F) \otimes \mathbb{K}$ such that $\mathrm{FK}_{\mathcal{R}}(\mathcal{P} ; \Phi)=$ $\operatorname{FK}_{\mathcal{R}}^{s}(I, W)$.

Proof. Throughout the proof, $\pi_{G}$ will denote the canonical surjective homomorphism from $\mathcal{T}(G)$ to $C^{*}(G)$, where $G$ is a graph. Also, $q_{w}^{G}$ and $t_{e}^{G}$ will denote the canonical generators of $\mathcal{T}(G)$ while $p_{w}^{G}$ and $s_{e}^{G}$ denote the canonical generators of $C^{*}(G)$. See Section 5 for more details and notation.

Part one: We first prove the row addition part of the proposition. Let $f \in$ $s^{-1}(u) \cap r^{-1}(v)$ which exists since $\mathrm{A}_{E}(u, v)>0$. Note that $F$ can be described as follows: $F^{0}=E^{0}$,

$$
F^{1}=\left(E^{1} \backslash\{f\}\right) \sqcup\left\{\bar{e}: e \in s_{E}^{-1}(v)\right\}
$$

where $s_{F}(e)=s_{E}(e)$ and $r_{F}(e)=r_{E}(e)$ for all $e \in E^{1} \backslash\{f\}, s_{F}(\bar{e})=u$ and $r_{F}(\bar{e})=r_{E}(e)$.

We now define a Toeplitz-Cuntz-Krieger $F$-family in $\mathcal{T}(E)$. Set $Q_{w}=q_{w}^{E}$ and

$$
T_{g}= \begin{cases}t_{e}^{E} & \text { if } g=e \in E^{1} \backslash\{f\}, \\ t_{f e}^{E} & \text { if } g=\bar{e} \text { for some } e \in s_{E}^{-1}(v) .\end{cases}
$$

A computation shows that $\left\{Q_{w}, T_{g}: w \in F^{0}, g \in F^{1}\right\}$ is a Toeplitz-Cuntz-Krieger $F$-family such that

$$
Q_{w}-\sum_{g \in s_{F}^{-1}(w)} T_{g} T_{g}^{*} \in \operatorname{ker}\left(\pi_{E}\right)
$$

for all $w \in F_{\text {reg. }}^{0}$. It follows that there exists a ${ }^{*}$-homomorphism $\Psi: \mathcal{T}(F) \rightarrow \mathcal{T}(E)$ such that $\Psi\left(q_{w}^{F}\right)=Q_{w}$ and $\Psi\left(t_{g}^{F}\right)=T_{g}$. Equation (6.1) implies that there exists a (unique) ${ }^{*}$-homomorphism $\bar{\Psi}: C^{*}(F) \rightarrow C^{*}(E)$ such that $\pi_{E} \circ \Psi=\bar{\Psi} \circ \pi_{F}$. 
Note that the identity map on $E^{0}=F^{0}$ induces a lattice isomorphism between the lattice of saturated hereditary subsets of $F^{0}$ and the lattice of saturated hereditary subsets of $E^{0}$. Since

$$
\bar{\Psi}\left(p_{w}^{F}\right)=\left(\bar{\Psi} \circ \pi_{F}\right)\left(q_{w}^{F}\right)=\left(\pi_{E} \circ \Psi\right)\left(q_{w}^{F}\right)=\pi_{E}\left(Q_{w}\right)=\pi_{E}\left(q_{w}^{E}\right)=p_{w}^{E},
$$

$\bar{\Psi}\left(\mathfrak{J}_{H}^{F}\right) \subseteq \mathfrak{J}_{H}^{E}$ for all saturated hereditary subsets $H$ of $F^{0}$. We have just shown that $\bar{\Psi}$ is a $\mathcal{P}$-equivariant homomorphism.

By Szy02, Theorem 1.2], $\bar{\Psi}$ is injective. Note that the only generator of $C^{*}(F)$ that is not in the image of $\bar{\Psi}$ is $s_{f}^{E}$. Since

$$
s_{f}^{E}=s_{f}^{E} p_{v}^{E}=s_{f}^{E} \sum_{e \in s_{E}^{-1}(v)} s_{e}^{E}\left(s_{e}^{E}\right)^{*}=\sum_{e \in s_{E}^{-1}(v)} \pi_{E}\left(T_{\bar{e}} T_{e}^{*}\right) \in \bar{\Psi}\left(C^{*}(F)\right),
$$

it follows that $\bar{\Psi}$ is surjective. Therefore, $\bar{\Psi}\left(\mathfrak{J}_{H}^{F}\right)=\mathfrak{J}_{H}^{E}$ for all saturated hereditary subsets $H$ of $F^{0}$, and, consequently $\bar{\Psi}$ is a $\mathcal{P}$-equivariant isomorphism (since $\bar{\Psi}$ is a ${ }^{*}$-isomorphism).

Let $\Phi: C^{*}(E) \rightarrow C^{*}(F)$ be the inverse of $\bar{\Psi}$. To prove that $\mathrm{FK}_{\mathcal{R}}(\mathcal{P} ; \Phi)=$ $\mathrm{FK}_{\mathcal{R}}\left(W^{\bullet}, I\right)$, it is enough to prove that $\mathrm{FK}_{\mathcal{R}}(\mathcal{P} ; \bar{\Psi})=\mathrm{FK}_{\mathcal{R}}\left(\left(W^{\bullet}\right)^{-1}, I\right)$.

Since $\bar{\Psi}\left(p_{w}^{F}\right)=p_{w}^{E}, \bar{\Psi}$ sends $\left[p_{w}^{F}\right]_{0} \in K_{0}\left(\mathfrak{J}_{H}^{F}\right)$ to $\left[p_{w}^{E}\right]_{0} \in K_{0}\left(\mathfrak{J}_{H}^{E}\right)$ which implies that $\bar{\Psi}$ induces the desired maps on $K_{0}$.

Now we want to show that $\bar{\Psi}$ induces the desired maps on $K_{1}$ (of the gauge simple subquotients of $C^{*}(F)$ ). For all components that do not contain both $u$ and $v$, it is clear that this holds (since both maps are clearly the identity map). In the opposite direction, if $u$ and $v$ both belong to a component, we only have to restrict to the subgraph of that component. For notational convenience, we show this for $C^{*}(E)$ instead, corresponding to the gauge simple case - the general case is analogous. Let $\mathbf{x}=\left(x_{w}\right)_{w \in F_{\mathrm{reg}}^{0}} \in \operatorname{ker}\left(\left(\mathrm{B}_{F}^{\bullet}\right)^{\top}\right)$. Note that

$$
\left(W^{\bullet}\right)^{\top}(\mathbf{x})=\sum_{\substack{x_{w} \neq 0 \\ w \neq v}} x_{w} e_{w}+\left(x_{v}+x_{u}\right) e_{v}
$$

In $K_{0}\left(\operatorname{ker}\left(\pi_{E}\right)\right)$,

$$
\begin{aligned}
& \sum_{x_{w} \neq 0} x_{w}\left[\Psi\left(q_{w}^{F}\right)-\sum_{g \in s_{F}^{-1}(w)} \Psi\left(t_{g}^{F}\right) \Psi\left(t_{g}^{F}\right)^{*}\right]_{0} \\
& =\sum_{\substack{x_{w} \neq 0 \\
w \neq u}} x_{w}\left[q_{w}^{E}-\sum_{e \in s_{E}^{-1}(w)} t_{e}^{E}\left(t_{e}^{E}\right)^{*}\right]_{0} \\
& \quad+x_{u}\left[q_{u}^{E}-\sum_{e \in s_{E}^{-1}(u) \backslash\{f\}} t_{e}^{E}\left(t_{e}^{E}\right)^{*}-\sum_{e \in s_{E}^{-1}(v)} t_{f e}^{E}\left(t_{f e}^{E}\right)^{*}\right]_{0} .
\end{aligned}
$$


Since $v$ is regular, $q_{v}^{E}-\sum_{e \in s_{E}^{-1}(v)} t_{e}^{E}\left(t_{e}^{E}\right)^{*} \in \operatorname{ker}\left(\pi_{E}\right)$. Hence, in $K_{0}\left(\operatorname{ker}\left(\pi_{E}\right)\right)$,

$$
\begin{aligned}
& {\left[q_{u}^{E}-\sum_{e \in s_{E}^{-1}(u) \backslash\{f\}} t_{e}^{E}\left(t_{e}^{E}\right)^{*}-\sum_{e \in s_{E}^{-1}(v)} t_{f e}^{E}\left(t_{f e}^{E}\right)^{*}\right]_{0}} \\
& \quad=\left[q_{u}^{E}-\sum_{e \in s_{E}^{-1}(u)} t_{e}^{E}\left(t_{e}^{E}\right)^{*}\right]_{0}+\left[t_{f}^{E}\left(q_{v}^{E}-\sum_{e \in s_{E}^{-1}(v)} t_{e}^{E}\left(t_{e}^{E}\right)^{*}\right)\left(t_{f}^{E}\right)^{*}\right]_{0} \\
& =\left[q_{u}^{E}-\sum_{e \in s_{E}^{-1}(u)} t_{e}^{E}\left(t_{e}^{E}\right)^{*}\right]_{0}+\left[q_{v}^{E}-\sum_{e \in s_{E}^{-1}(v)} t_{e}^{E}\left(t_{e}^{E}\right)^{*}\right]_{0} .
\end{aligned}
$$

Thus,

$$
\begin{aligned}
& \sum_{x_{w} \neq 0} x_{w}\left[\Psi\left(q_{w}^{F}\right)-\sum_{g \in s_{F}^{-1}(w)} \Psi\left(t_{g}^{F}\right) \Psi\left(t_{g}^{F}\right)^{*}\right]_{0} \\
& =\sum_{\substack{x_{w} \neq 0 \\
w \neq u}} x_{w}\left[q_{w}^{E}-\sum_{e \in s_{E}^{-1}(w)} t_{e}^{E}\left(t_{e}^{E}\right)^{*}\right]_{0} \\
& \quad+x_{u}\left[q_{u}^{E}-\sum_{e \in s_{E}^{-1}(u)} t_{e}^{E}\left(t_{e}^{E}\right)^{*}\right]_{0}+x_{u}\left[q_{v}^{E}-\sum_{e \in s_{E}^{-1}(v)} t_{e}^{E}\left(t_{e}^{E}\right)^{*}\right]_{0} .
\end{aligned}
$$

So,

$$
\begin{aligned}
\left(\kappa_{E} \circ \partial_{1}^{E} \circ K_{1}(\bar{\Psi}) \circ \chi_{1}^{F}\right)(\mathbf{x}) & =\left(\kappa_{E} \circ K_{0}(\Psi) \circ \partial_{1}^{F} \circ \chi_{1}^{F}\right)(\mathbf{x}) \\
= & \sum_{\substack{x_{w} \neq 0 \\
w \neq u}} x_{w} \kappa_{E}\left(\left[q_{w}^{E}-\sum_{e \in s_{E}^{-1}(w)} t_{e}^{E}\left(t_{e}^{E}\right)^{*}\right]_{0}\right) \\
& +x_{u} \kappa_{E}\left(\left[q_{u}^{E}-\sum_{e \in s_{E}^{-1}(u)} t_{e}^{E}\left(t_{e}^{E}\right)^{*}\right]_{0}\right)+x_{u} \kappa_{E}\left(\left[q_{v}^{E}-\sum_{e \in s_{E}^{-1}(v)} t_{e}^{E}\left(t_{e}^{E}\right)^{*}\right]_{0}\right) \\
= & \sum_{\substack{x_{w} \neq 0 \\
w \neq u}} x_{w} e_{w}+x_{u} e_{u}+x_{u} e_{v} \\
= & \sum_{\substack{x_{w} \neq 0 \\
w \neq v}} x_{w} e_{w}+\left(x_{u}+x_{v}\right) e_{v} .
\end{aligned}
$$

Hence, $\bar{\Psi}$ induces the desired maps on $K_{1}$.

Part two: We now prove the column addition part of the proposition. Note that $F$ can be described as follows: Take an edge $f$ from $u$ to $v$. Set $F^{0}=E^{0}$,

$$
F^{1}=\left(E^{1} \backslash\{f\}\right) \sqcup\left\{\bar{e}: e \in r_{E}^{-1}(u)\right\}
$$


with $s_{F}(e)=s_{E}(e), r_{F}(e)=r_{E}(e)$ for all $e \in E^{1} \backslash\{f\}$, and $s_{F}(\bar{e})=s_{E}(e)$ and $r_{F}(\bar{e})=v$.

We define a Toeplitz-Cuntz-Krieger $F$-family in $\mathcal{T}(E)$. Set

$$
Q_{w}= \begin{cases}q_{w}^{E} & \text { if } w \neq u, \\ q_{u}^{E}-t_{f}^{E}\left(t_{f}^{E}\right)^{*} & \text { if } w=u,\end{cases}
$$

and

$$
T_{g}= \begin{cases}t_{g}^{E} & \text { if } g \in E^{1} \backslash\{f\} \text { and } r_{E}(g) \neq u, \\ t_{g}^{E} Q_{u} & \text { if } g \in E^{1} \backslash\{f\} \text { and } r_{E}(g)=u, \\ t_{e f}^{E} & \text { if } g=\bar{e} \text { for some } e \in r_{E}^{-1}(u) .\end{cases}
$$

A computation shows that $\left\{Q_{w}, T_{g}: w \in F^{0}, g \in F^{1}\right\}$ is a Toeplitz-Cuntz-Krieger $F$-family in $\mathcal{T}(E)$ such that

$$
Q_{w}-\sum_{g \in s_{F}^{-1}(w)} T_{g} T_{g}^{*} \in \operatorname{ker}\left(\pi_{E}\right)
$$

for all $w \in F_{\text {reg. }}^{0}$. It follows that there exists a ${ }^{*}$-homomorphism $\Psi: \mathcal{T}(F) \rightarrow \mathcal{T}(E)$ such that $\Psi\left(q_{w}^{F}\right)=Q_{w}$ and $\Psi\left(t_{g}^{F}\right)=T_{g}$. Equation (6.2) implies that there exists a (unique) *-homomorphism $\bar{\Psi}: C^{*}(F) \rightarrow C^{*}(E)$ such that $\pi_{E} \circ \Psi=\bar{\Psi} \circ \pi_{F}$. Moreover, $\bar{\Psi}\left(C^{*}(F)\right)=P C^{*}(E) P$, where $P=\sum_{w \in E^{0}} \pi_{E}\left(Q_{w}\right)$. Note that $P C^{*}(E) P$ is full in $C^{*}(E)$. Also, by Szy02, Theorem 1.2], $\bar{\Psi}$ is injective. Hence, $\bar{\Psi}: C^{*}(F) \rightarrow$ $P C^{*}(E) P$ is a *-isomorphism.

Since $P C^{*}(E) P$ is full in $C^{*}(E)$, it follows from Bro77, Corollary 2.6 and its proof] that there exists $V$ in the multiplier algebra of $C^{*}(E) \otimes \mathbb{K}$ such that $V^{*} V=$ $P \otimes 1_{\mathbb{B}\left(\ell^{2}\right)}$ and $V V^{*}=1_{\mathcal{M}\left(C^{*}(E)\right)} \otimes 1_{\mathbb{B}\left(\ell^{2}\right)}$. Then $\operatorname{Ad}(V) \circ\left(\bar{\Psi} \otimes \operatorname{id}_{\mathbb{K}}\right): C^{*}(F) \otimes \mathbb{K} \rightarrow$ $C^{*}(E) \otimes \mathbb{K}$ is a ${ }^{*}$-isomorphism.

We will show that $\operatorname{Ad}(V) \circ\left(\bar{\Psi} \otimes \mathrm{id}_{\mathbb{K}}\right)$ is a $\mathcal{P}$-equivariant isomorphism. We first show that $\bar{\Psi}$ is a $\mathcal{P}$-equivariant homomorphism, which implies that $\operatorname{Ad}(V) \circ\left(\bar{\Psi} \otimes \operatorname{id}_{\mathbb{K}}\right)$ is a $\mathcal{P}$-equivariant homomorphism. Note that the identity map on $E^{0}=F^{0}$ induces a lattice isomorphism between the lattice of saturated hereditary subsets of $F^{0}$ and the lattice of saturated hereditary subsets of $E^{0}$. Let $H$ be a hereditary subset of $F^{0}$. Let $w \in H$. Since $\bar{\Psi}\left(p_{w}^{F}\right) \leq p_{w}^{E}$, we have that $\bar{\Psi}\left(p_{w}^{F}\right) \in \mathfrak{J}_{H}^{E}$. Hence, $\bar{\Psi}\left(\mathfrak{J}_{H}^{F}\right) \subseteq \mathfrak{J}_{H}^{E}$. We have just shown that $\bar{\Psi}$ is a $\mathcal{P}$-equivariant homomorphism.

Let $H$ be a saturated hereditary subset of $F^{0}$. Then $\operatorname{Ad}(V) \circ\left(\bar{\Psi} \otimes \operatorname{id}_{\mathbb{K}}\right)\left(\mathfrak{J}_{H}^{F} \otimes \mathbb{K}\right)$ is the ideal of $C^{*}(E) \otimes \mathbb{K}$ generated by $\left\{V\left(\pi_{E}\left(Q_{w}\right) \otimes e_{11}\right) V^{*} \mid w \in H\right\}$, where $e_{11}$ is a minimal nonzero projection in $\mathbb{K}$. Since $V\left(\pi_{E}\left(Q_{w}\right) \otimes e_{11}\right) V^{*}$ is Murrayvon Neumann equivalent to $\pi_{E}\left(Q_{w}\right) \otimes e_{11}$ for all $w \in H$, we have that $\operatorname{Ad}(V) \circ$ $\left(\bar{\Psi} \otimes \mathrm{id}_{\mathbb{K}}\right)\left(\mathfrak{J}_{H}^{F} \otimes \mathbb{K}\right)$ is the ideal of $C^{*}(E) \otimes \mathbb{K}$ generated by $\left\{\pi_{E}\left(Q_{w}\right) \otimes e_{11} \mid w \in H\right\}$. Note that

$$
p_{u}^{E} \otimes e_{11}=\left(p_{u}^{E}-s_{f}^{E}\left(s_{f}^{E}\right)^{*}\right) \otimes e_{11}+\left(s_{f}^{E}\left(s_{f}^{E}\right)^{*}\right) \otimes e_{11}
$$

and $\left(s_{f}^{E}\left(s_{f}^{E}\right)^{*}\right) \otimes e_{11}$ is Murray-von Neumann equivalent to $p_{v}^{E} \otimes e_{22}$, where $e_{22}$ is a minimal nonzero projection in $\mathbb{K}$ that is orthogonal to $e_{11}$. Hence, $p_{u}^{E} \otimes e_{11}$ is Murray-von Neumann equivalent to $\left(p_{u}^{E}-s_{f}^{E}\left(s_{f}^{E}\right)^{*}\right) \otimes e_{11}+p_{v}^{E} \otimes e_{22}$ which is in the ideal of $C^{*}(E) \otimes \mathbb{K}$ generated by $\left\{\pi_{E}\left(Q_{w}\right) \otimes e_{11} \mid w \in H\right\}$ whenever $u \in$ $H$. Therefore, the ideal of $C^{*}(E) \otimes \mathbb{K}$ generated by $\left\{\pi_{E}\left(Q_{w}\right) \otimes e_{11} \mid w \in H\right\}$ is $\mathfrak{J}_{H}^{E} \otimes \mathbb{K}$. Thus, $\operatorname{Ad}(V) \circ\left(\bar{\Psi} \otimes \mathrm{id}_{\mathbb{K}}\right)\left(\mathfrak{J}_{H}^{F} \otimes \mathbb{K}\right)=\mathfrak{J}_{H}^{E} \otimes \mathbb{K}$. We have just shown that $\operatorname{Ad}(V) \circ\left(\bar{\Psi} \otimes \operatorname{id}_{\mathbb{K}}\right)$ is a $\mathcal{P}$-equivariant isomorphism. 
We now show that $\operatorname{Ad}(V) \circ\left(\bar{\Psi} \otimes \mathrm{id}_{\mathbb{K}}\right)$ induces $\operatorname{FK}_{\mathcal{R}}^{s}\left(I, W^{-1}\right)$. Since $V$ is an isometry in the multiplier algebra $\mathcal{M}\left(C^{*}(E) \otimes \mathbb{K}\right)$ of $C^{*}(E) \otimes \mathbb{K}$, it is enough to show that $\bar{\Psi}$ induces $\operatorname{FK}_{\mathcal{R}}\left(I, W^{-1}\right)$ ( $c f$. [RR07, Lemma 3.4]. Note that

$$
\left(W^{-1}\right)^{\top}\left(e_{w}\right)= \begin{cases}e_{w} & \text { if } w \neq u \\ e_{u}-e_{v} & \text { if } w=u\end{cases}
$$

Let $H$ be a saturated subset $H$ of $F^{0}$. It is trivial to check that the induced maps are equal on $K_{0}$ of $\mathfrak{J}_{H}^{E}$ whenever $u \notin H$. So assume that $u \in H$. Then $p_{u}^{E}, s_{f}^{E} \in \mathfrak{J}_{H}^{E}$, and, consequently, we have that

$$
\begin{aligned}
\sum_{w \in H} x_{w}\left[\bar{\Psi}\left(p_{w}^{F}\right)\right]_{0} & =\sum_{w \in H \backslash\{u\}} x_{w}\left[p_{w}^{E}\right]_{0}+x_{u}\left[p_{u}^{E}-s_{f}^{E}\left(s_{f}^{E}\right)^{*}\right]_{0} \\
& =\sum_{w \in H \backslash\{u\}} x_{w}\left[p_{w}^{E}\right]_{0}+x_{u}\left[p_{u}^{E}\right]_{0}-x_{u}\left[s_{f}^{E}\left(s_{f}^{E}\right)^{*}\right]_{0} \\
& =\sum_{w \in H \backslash\{u\}} x_{w}\left[p_{w}^{E}\right]_{0}+x_{u}\left[p_{u}^{E}\right]_{0}-x_{u}\left[\left(s_{f}^{E}\right)^{*} s_{f}^{E}\right]_{0} \\
& =\sum_{w \in H \backslash\{u\}} x_{w}\left[p_{w}^{E}\right]_{0}+x_{u}\left[p_{u}^{E}\right]_{0}-x_{u}\left[p_{r_{E}}^{E}(f)\right]_{0} \\
& =\sum_{w \in H \backslash\{u\}} x_{w}\left[p_{w}^{E}\right]_{0}+x_{u}\left[p_{u}^{E}\right]_{0}-x_{u}\left[p_{v}^{E}\right]_{0}
\end{aligned}
$$

in $K_{0}\left(\mathfrak{J}_{H}^{E}\right)$. So,

$$
\left(K_{0}(\bar{\Psi}) \circ \chi_{0}^{H}\right)\left(\sum_{w \in H} x_{w} \bar{e}_{w}\right)=\sum_{w \in H} x_{w}\left[\bar{\Psi}\left(p_{w}^{F}\right)\right]_{0}=\left(\chi_{0}^{H} \circ\left(W^{-1}\right)^{\top}\right)\left(\sum_{w \in H} x_{w} \bar{e}_{w}\right)
$$

in $K_{0}\left(\mathfrak{J}_{H}^{E}\right)$. Thus, $\bar{\Psi}$ induces the desired maps on $K_{0}$.

We now show that $\bar{\Psi}$ induces the desired maps on $K_{1}$. Let $w \in F_{\text {reg }}^{0}=E_{\text {reg }}^{0}$ and $w \neq u$. Then

$$
\begin{aligned}
& \Psi\left(q_{w}^{F}\right)-\sum_{g \in s_{F}^{-1}(w)} \Psi\left(t_{g}^{F}\left(t_{g}^{F}\right)^{*}\right) \\
& =q_{w}^{E}-\sum_{\substack{e \in s_{E}^{-1}(w) \\
r_{E}(e) \neq u}} t_{e}^{E}\left(t_{e}^{E}\right)^{*}-\sum_{\substack{e \in s_{E}^{-1}(w) \\
r_{E}(e)=u}} t_{e}^{E}\left(q_{u}^{E}-t_{f}^{E}\left(t_{f}^{E}\right)^{*}\right)\left(t_{e}^{E}\right)^{*} \\
& -\sum_{\substack{e \in s_{E}^{-1}(w) \\
r_{E}(e)=u}} t_{e}^{E} t_{f}^{E}\left(t_{f}^{E}\right)^{*}\left(t_{e}^{E}\right)^{*} \\
& =q_{w}^{E}-\sum_{\substack{e \in s_{E}^{-1}(w) \\
r_{E}(e) \neq u}} t_{e}^{E}\left(t_{e}^{E}\right)^{*}-\sum_{\substack{e \in s_{E}^{-1}(w) \\
r_{E}(e)=u}} t_{e}^{E}\left(t_{e}^{E}\right)^{*} \\
& =q_{w}^{E}-\sum_{e \in s_{E}^{-1}(w)} t_{e}^{E}\left(t_{e}^{E}\right)^{*} .
\end{aligned}
$$


If $u$ is regular, then

$$
\begin{aligned}
\Psi\left(q_{u}^{F}\right)- & \sum_{g \in s_{F}^{-1}(u)} \Psi\left(t_{g}^{F}\left(t_{g}^{F}\right)^{*}\right) \\
= & q_{u}^{E}-t_{f}^{E}\left(t_{f}^{E}\right)^{*}-\sum_{\substack{e \in\left(s_{E}^{-1}(u) \backslash\{f\}\right) \\
r_{E}(e) \neq u}} t_{e}^{E}\left(t_{e}^{E}\right)^{*}-\sum_{\substack{e \in s_{E}^{-1}(u) \\
r_{E}(e)=u}} t_{e}^{E}\left(q_{u}^{E}-t_{f}^{E}\left(t_{f}^{E}\right)^{*}\right)\left(t_{e}^{E}\right)^{*} \\
& -\sum_{\substack{e \in s_{E}^{-1}(u) \\
r_{E}(e)=u}} t_{e}^{E} t_{f}^{E}\left(t_{f}^{E}\right)^{*}\left(t_{e}^{E}\right)^{*} \\
= & q_{u}^{E}-\sum_{\substack{e \in s_{E}^{-1}(u) \\
r_{E}(e) \neq u}} t_{e}^{E}\left(t_{e}^{E}\right)^{*}-\sum_{\substack{e \in s_{E}^{-1}(u) \\
r_{E}(e)=u}} t_{e}^{E}\left(t_{e}^{E}\right)^{*} \\
= & q_{u}^{E}-\sum_{e \in s_{E}^{-1}(u)} t_{e}^{E}\left(t_{e}^{E}\right)^{*} .
\end{aligned}
$$

Now we want to show that $\bar{\Psi}$ induces the desired maps on $K_{1}$ (of the gauge simple subquotients of $\left.C^{*}(E)\right)$. For all components that do not contain both $u$ and $v$, it is clear that this holds (since both maps are clearly the identity map). In the opposite direction, if $u$ and $v$ both belong to a component, we only have to restrict to the subgraph of that component. For notational convenience, we show this for $C^{*}(E)$ instead, corresponding to the gauge simple case - the general case is analogous. Let $\mathbf{x}=\left(x_{w}\right)_{w \in F_{\text {reg }}^{0}} \in \operatorname{ker}\left(\left(\mathrm{B}_{F}^{\bullet}\right)^{\top}\right)$. Then, in $K_{0}\left(\operatorname{ker}\left(\pi_{E}\right)\right)$, we have that

$$
\sum_{x_{w} \neq 0} x_{w}\left[\Psi\left(q_{w}^{F}\right)-\sum_{e \in s_{F}^{-1}(w)} \Psi\left(t_{e}^{F}\left(t_{e}^{F}\right)^{*}\right)\right]_{0}=\sum_{x_{w} \neq 0} x_{w}\left[q_{w}^{E}-\sum_{e \in s_{E}^{-1}(w)} t_{e}^{E}\left(t_{e}^{E}\right)^{*}\right]_{0} .
$$

So,

$$
\begin{aligned}
\left(\kappa_{E} \circ \partial_{1}^{E} \circ K_{1}(\bar{\Psi}) \circ \chi_{1}^{F}\right)(\mathbf{x}) & =\left(\kappa_{E} \circ K_{0}(\Psi) \circ \partial_{1}^{F} \circ \chi_{1}^{F}\right)(\mathbf{x}) \\
& =\kappa_{E}\left(\sum_{x_{w} \neq 0} x_{w}\left[q_{w}^{E}-\sum_{e \in s_{E}^{-1}(w)} t_{e}^{E}\left(t_{e}^{E}\right)^{*}\right]_{0}\right) \\
& =\sum_{x_{w} \neq 0} x_{w} e_{w} .
\end{aligned}
$$

Hence, $\bar{\Psi}$ induces the desired maps on $K_{1}$.

Set $\Phi=\left(\operatorname{Ad}(V) \circ\left(\bar{\Psi} \otimes \operatorname{id}_{\mathbb{K}}\right)\right)^{-1}: C^{*}(E) \otimes \mathbb{K} \rightarrow C^{*}(F) \otimes \mathbb{K}$. Then $\Phi$ is a $\mathcal{P}$ equivariant isomorphism that induces $\operatorname{FK}_{\mathcal{R}}^{s}(I, W)$.

Recall from [ERRS16b, Definition 4.23] that when $E$ is a finite graph with no sinks and no sources satisfying Condition $(K)$, then we write $B_{E} \in \mathfrak{M}_{\mathcal{P}}^{+}(\mathbf{n}, \mathbb{Z})$ whenever $\mathrm{B}_{E} \in \mathfrak{M}_{\mathcal{P}}(\mathbf{n}, \mathbb{Z})$ and for all $i, j \in \mathcal{P}$

(i) if $i \preceq j$, then $\mathrm{B}_{E}\{i, j\}>0$,

(ii) $n_{i} \geq 3$ and the Smith normal form of $\mathrm{B}_{E}\{i\}$ has at least two 1's (and thus the rank of $\mathrm{B}_{E}\{i\}$ is at least 2). 
Remark 6.2. Let $E$ be a finite graph with no sinks and no sources satisfying Condition $(\mathrm{K})$ such that $\mathrm{B}_{E} \in \mathfrak{M}_{\mathcal{P}}^{+}(\mathbf{n}, \mathbb{Z})$. Then $\mathrm{B}_{E} \in \mathfrak{M}_{\mathcal{P}}^{\circ \circ}(\mathbf{n}, \mathbb{Z})$ and every vertex in $E$ emits at least two edges since the diagonal entries $\mathrm{A}_{E}=\mathrm{B}_{E}+I$ are greater than or equal to 2 .

Lemma 6.3. Let $A$ be a nondegenerate matrix that satisfies Condition (II). Then there exists a finite graph $E$ with no sinks and no sources that satisfies Condition $(\mathrm{K})$ such that $\mathcal{O}_{A} \otimes \mathbb{K} \cong C^{*}(E) \otimes \mathbb{K}$ and $\mathrm{B}_{E} \in \mathfrak{M}_{\mathcal{P}}^{+}(\mathbf{n}, \mathbb{Z})$, for a suitable $\mathbf{n}$ and $\mathcal{P}$.

Proof. The matrix $A$ defines a graph $F$ (with adjacency matrix $A$ ) with no sinks and no sources that satisfies Condition $(\mathrm{K})$. The graph $C^{*}$-algebra $C^{*}(F)$ is isomorphic to the Cuntz-Krieger algebra $\mathcal{O}_{A}$ via a ${ }^{*}$-isomorphism defined by $p_{i, F} \mapsto s_{i} s_{i}^{*}$ and $s_{e, F} \mapsto s_{s_{F}(e)} s_{r_{F}(e)} s_{r_{F}(e)}^{*}$, where $\left\{p_{i, F}, s_{e, F}\right\}$ is a Cuntz-Krieger $F$-family generating $C^{*}(F)$ and $\left\{s_{1}, \ldots, s_{n}\right\}$ is a universal family of partial isometries generating $\mathcal{O}_{A}$. Thus we can work with $F$ instead. Now it follows from [ERRS16b, Lemma 4.24] and ERRS16b, Theorem 2.20] that we can choose a finite graph $E$ such that $\mathrm{B}_{E} \in \mathfrak{M}_{\mathcal{P}}^{+}(\mathbf{m} \times \mathbf{n}, \mathbb{Z})$ and $\mathcal{O}_{A} \otimes \mathbb{K} \cong C^{*}(E) \otimes \mathbb{K}$. Since $\mathrm{B}_{E} \in \mathfrak{M}_{\mathcal{P}}^{+}(\mathbf{m} \times \mathbf{n}, \mathbb{Z})$, the graph $E$ does not have any source. Note that Condition $(K)$ with no sinks means exactly that the temperature is constant 1; cf. ERRS16b, Definition 4.16 and Lemma 4.20(i)]. Since the temperature is clearly a stable isomorphism invariant, it follows that $E$ has no sinks and satisfies Condition $(\mathrm{K})$, as well as $\mathbf{m}=\mathbf{n}$.

Lemma 6.4. Let $E$ be a finite graph with no sinks and no sources satisfying Condition $(\mathrm{K})$ such that $\mathrm{B}_{E} \in \mathfrak{M}_{\mathcal{P}}^{+}(\mathbf{n}, \mathbb{Z})$, and let $j \in \mathcal{P}$ be given. Then there exists a finite graph $F$ with no sinks and no sources satisfying Condition $(\mathrm{K})$ such that $\mathrm{B}_{F} \in \mathfrak{M}_{\mathcal{P}}^{+}\left(\mathbf{n}+\mathbf{e}_{j}, \mathbb{Z}\right)$, and such that there exists a $\mathcal{P}$-equivariant isomorphism $\Phi$ from $C^{*}(E) \otimes \mathbb{K}$ to $C^{*}(F) \otimes \mathbb{K}$ satisfying $\operatorname{FK}_{\mathcal{R}}(\mathcal{P} ; \Phi)=\mathrm{FK}_{\mathcal{R}}^{s}(U, V)$ for some $\mathrm{SL}_{\mathcal{P}}$ equivalence $(U, V)$ from $-\iota_{\mathbf{e}_{j}}\left(-\mathrm{B}_{E}\right)$ to $\mathrm{B}_{F}$.

Proof. Let $e_{0}$ be an edge that is part of a cycle that lies in the component corresponding to $j \in \mathcal{P}$. Let $E^{\prime}$ be the simple edge expansion graph at $e_{0}$, and let $v^{\prime}$ denote the new vertex ( $c f$. Definition [5.1). It follows from Proposition 5.4 that $\mathrm{B}_{E^{\prime}} \in \mathfrak{M}_{\mathcal{P}}^{\circ}\left(\mathbf{n}+\mathbf{e}_{j}, \mathbb{Z}\right)$ and that there exists a $\mathcal{P}$-equivariant isomorphism $\Phi^{\prime}$ from $C^{*}(E) \otimes \mathbb{K}$ to $C^{*}\left(E^{\prime}\right) \otimes \mathbb{K}$ such that $\mathrm{FK}_{\mathcal{R}}\left(\mathcal{P}, \Phi^{\prime}\right)=\mathrm{FK}_{\mathcal{R}}^{s}\left(U^{\prime}, V^{\prime}\right)$ for some $\mathrm{SL}_{\mathcal{P}}$-equivalence $\left(U^{\prime}, V^{\prime}\right)$ from $-\iota_{\mathbf{e}_{j}}\left(-\mathrm{B}_{E}\right)$ to $\mathrm{B}_{E^{\prime}}$. The idea is to use Proposition 6.1 to construct a finite graph $F$ with no sinks and no sources satisfying Condition $(\mathrm{K})$ such that $\mathrm{B}_{F} \in \mathfrak{M}_{\mathcal{P}}^{+}\left(\mathbf{n}+\mathbf{e}_{j}, \mathbb{Z}\right)$, and a $\mathcal{P}$-equivariant isomorphism $\Phi^{\prime \prime}$ from $C^{*}\left(E^{\prime}\right) \otimes \mathbb{K}$ to $C^{*}(F) \otimes \mathbb{K}$ such that $\mathrm{FK}_{\mathcal{R}}\left(\mathcal{P}, \Phi^{\prime \prime}\right)=\mathrm{FK}_{\mathcal{R}}^{s}\left(U^{\prime \prime}, V^{\prime \prime}\right)$ for some $\mathrm{SL}_{\mathcal{P}}$-equivalence $\left(U^{\prime \prime}, V^{\prime \prime}\right)$ from $\mathrm{B}_{E^{\prime}}$ to $\mathrm{B}_{F}$. We have that $\mathrm{B}_{E^{\prime}} \in \mathfrak{M}_{\mathcal{P}}^{\circ}\left(\mathbf{n}+\mathbf{e}_{j}, \mathbb{Z}\right)$, and since $E$ has no transition states, no cyclic components, no sinks and no sources, it follows that $E^{\prime}$ has no transition states, no cyclic components, no sinks and no sources, and thus that $\mathrm{B}_{E^{\prime}} \in \mathfrak{M}_{\mathcal{P}}^{\circ \circ}\left(\mathbf{n}+\mathbf{e}_{j}, \mathbb{Z}\right)$. We may assume that $v_{0}=s\left(e_{0}\right)$ corresponds to the last row of the diagonal block $\mathrm{A}\{j\}$. Let $v_{1}=r\left(e_{0}\right)$. We will now perform a series of column and row additions on the matrix $A_{E^{\prime}}-I$ according to Proposition 6.1. Using a column addition within block $j$, we can make sure that $v_{0}$ supports at least two loops. Adding column $v_{0}$ to $v^{\prime}$, we get all entries of the column $v^{\prime}$ in $j$ th diagonal block to be positive except for the last entry. Adding row $v_{1}$ twice to row $v^{\prime}$, the $j$ th diagonal block is positive. Now we can use column additions and row additions to get all the off-diagonal blocks that are not forced by the order on $\mathcal{P}$ to be zero to be positive, and we thus end up with a graph $F$ such that $\mathrm{B}_{F} \in \mathfrak{M}_{\mathcal{P}}^{+}\left(\mathbf{n}+\mathbf{e}_{j}, \mathbb{Z}\right)$. Since these are all legal column and row operations according to Proposition 6.1 we get the desired result from Proposition 5.4 


\section{Cuntz SPlice implies Stable isomorphism}

In ERRS16a, it was proved that Cuntz splicing a graph gives rise to a graph whose $C^{*}$-algebra is stably isomorphic to the $C^{*}$-algebra of the original graph (generalizing a result in Res06]). This is used for instance in the proofs of [ERRS16b] to get a classification result. As we are proving a strong classification result, we will need to know more about what this stable isomorphism induces on the reduced filtered $K$-theory. This will be the main aim of this section. In the end of the section, we will use the results to stitch together the proofs of the main results.

We recall some notation from ERRS16c that will be used throughout the rest of this section.

Notation 7.1. Let $\mathbf{E}_{*}$ and $\mathbf{E}_{* *}$ denote the graphs:
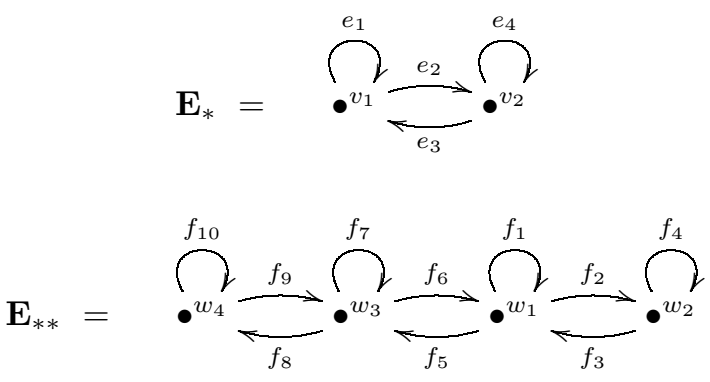

The graph $\mathbf{E}_{*}$ is what we attach when we Cuntz splice. If we instead attach the graph $\mathbf{E}_{* *}$, we Cuntz spliced twice.

Let $E=\left(E^{0}, E^{1}, r_{E}, s_{E}\right)$ be a graph and let $u$ be a vertex of $E$. Then $E_{u,-}$ can be described as follows (up to canonical isomorphism):

$$
\begin{aligned}
& E_{u,-}^{0}=E^{0} \sqcup \mathbf{E}_{*}^{0}, \\
& E_{u,-}^{1}=E^{1} \sqcup \mathbf{E}_{*}^{1} \sqcup\left\{d_{1}, d_{2}\right\}
\end{aligned}
$$

with $\left.r_{E_{u,-}}\right|_{E^{1}}=r_{E},\left.s_{E_{u,-}}\right|_{E^{1}}=s_{E},\left.r_{E_{u,-}}\right|_{\mathbf{E}_{*}^{1}}=r_{\mathbf{E}_{*}},\left.s_{E_{u,-}}\right|_{\mathbf{E}_{*}^{1}}=s_{\mathbf{E}_{*}}$, and

$$
\begin{aligned}
& s_{E_{u,-}}\left(d_{1}\right)=u, \quad r_{E_{u,-}}\left(d_{1}\right)=v_{1}, \\
& s_{E_{u,-}}\left(d_{2}\right)=v_{1}, \quad r_{E_{u,-}}\left(d_{2}\right)=u \text {. }
\end{aligned}
$$

Moreover, $E_{u,--}$ can be described as follows (up to canonical isomorphism):

$$
\begin{aligned}
& E_{u,--}^{0}=E^{0} \sqcup \mathbf{E}_{* *}^{0}, \\
& E_{u,--}^{1}=E^{1} \sqcup \mathbf{E}_{* *}^{1} \sqcup\left\{d_{1}, d_{2}\right\}
\end{aligned}
$$

with $\left.r_{E_{u,--}}\right|_{E^{1}}=r_{E},\left.s_{E_{u,--}}\right|_{E^{1}}=s_{E},\left.r_{E_{u,--}}\right|_{\mathbf{E}_{* *}^{1}}=r_{\mathbf{E}_{* *}},\left.s_{E_{u,--}}\right|_{\mathbf{E}_{* *}^{1}}=s_{\mathbf{E}_{* *}}$, and

$$
\begin{array}{ll}
s_{E_{u,--}}\left(d_{1}\right)=u, & r_{E_{u,--}}\left(d_{1}\right)=w_{1}, \\
s_{E_{u,--}}\left(d_{2}\right)=w_{1}, & r_{E_{u,--}}\left(d_{2}\right)=u .
\end{array}
$$

Proposition 7.2. Let $E$ be a finite graph with no sinks and no sources satisfying Condition $(\mathrm{K})$ such that $\mathrm{B}_{E} \in \mathfrak{M}_{\mathcal{P}}^{+}(\mathbf{n}, \mathbb{Z})$. Let $u$ be a vertex in $E^{0}$, and assume that it belongs to the component corresponding to the block $j \in \mathcal{P}$. Then $\mathrm{B}_{E_{u,--}}$ is an element of $\mathfrak{M}_{\mathcal{P}}\left(\mathbf{n}+4 \mathbf{e}_{j}, \mathbb{Z}\right)$, and there are an $\mathrm{SL}_{\mathcal{P}}$-equivalence $(U, V)$ from $-\iota_{4 \mathbf{e}_{j}}\left(-\mathrm{B}_{E}\right)$ to $\mathrm{B}_{E_{u},-}$ and a $\mathcal{P}$-equivariant isomorphism $\Phi$ from $C^{*}(E) \otimes \mathbb{K}$ to $C^{*}\left(E_{u,--}\right) \otimes \mathbb{K}$ such that $\mathrm{FK}_{\mathcal{R}}(\mathcal{P} ; \Phi)=\mathrm{FK}_{\mathcal{R}}^{s}(U, V)$. 
Proof. The $j$ th diagonal block of $\mathrm{B}_{E_{u,--}}$ can be described as the matrix

$$
\left(\begin{array}{cc}
\mathrm{B}_{E}\{j\} & \left(\begin{array}{cccc}
0 & 0 & 0 & 0 \\
\vdots & \vdots & \vdots & \vdots \\
1 & 0 & 0 & 0
\end{array}\right) \\
\left(\begin{array}{cccc}
0 & \cdots & 1 \\
0 & \cdots & 0 \\
0 & \cdots & 0 \\
0 & \cdots & 0
\end{array}\right) & \left(\begin{array}{llll}
0 & 1 & 1 & 0 \\
1 & 0 & 0 & 0 \\
1 & 0 & 0 & 1 \\
0 & 0 & 1 & 0
\end{array}\right)
\end{array}\right) .
$$

Let $U, V \in \mathrm{SL}_{\mathcal{P}}\left(\mathbf{n}+4 \mathbf{e}_{j}, \mathbb{Z}\right)$ be the identity matrices everywhere except for the $j$ th diagonal block where they are

$$
\begin{aligned}
& \left(\begin{array}{cc}
I & \left(\begin{array}{cccc}
0 & 0 & 0 & 0 \\
\vdots & \vdots & \vdots & \vdots \\
0 & 1 & 0 & 0
\end{array}\right) \\
\left(\begin{array}{ccc}
0 & \cdots & 0 \\
0 & \cdots & 0 \\
0 & \cdots & 0 \\
0 & \cdots & 0
\end{array}\right) & \left(\begin{array}{llll}
1 & 0 & 0 & 1 \\
0 & 1 & 0 & 0 \\
0 & 0 & 1 & 0 \\
0 & 0 & 0 & 1
\end{array}\right)
\end{array}\right)
\end{aligned}
$$

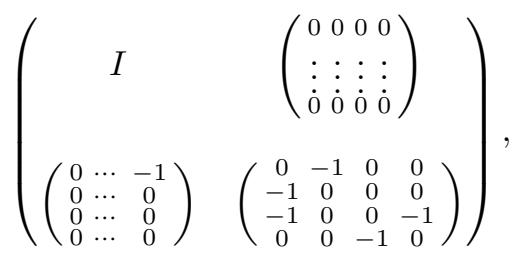

respectively. It is easy to verify that these blocks have indeed determinant 1 and that $(U, V)$ is an $\mathrm{SL}_{\mathcal{P}}$-equivalence from $-\iota_{4 \mathbf{e}_{j}}\left(-\mathrm{B}_{E}\right)$ to $\mathrm{B}_{E_{u,--}}$. The idea now is to deduce the existence of $\Phi$ from [Boy02, Theorem 4.4] and Proposition [6.1, but $E$ and $E_{u,--}$ do not satisfy the assumption of Boy02, Theorem 4.4]. We therefore make a small detour. By using column and row additions in the same way as in the proof of Lemma 6.4 together with Proposition 6.1 we see that we get two finite graphs $F$ and $F^{\prime}$ satisfying Condition $(\mathrm{K})$ with no sinks and no sources such that $\mathrm{B}_{F}, \mathrm{~B}_{F^{\prime}} \in \mathfrak{M}_{\mathcal{P}}^{+}\left(\mathbf{n}+4 \mathbf{e}_{j}, \mathbb{Z}\right)$ and such that there exist $\mathcal{P}$-equivariant isomorphisms $\Psi$ from $C^{*}(E) \otimes \mathbb{K}$ to $C^{*}(F) \otimes \mathbb{K}$ and $\Psi^{\prime}$ from $C^{*}\left(E_{u,--}\right) \otimes \mathbb{K}$ to $C^{*}\left(F^{\prime}\right) \otimes \mathbb{K}$ with $\mathrm{FK}_{\mathcal{R}}(\mathcal{P} ; \Psi)=\mathrm{FK}_{\mathcal{R}}^{s}\left(U_{1}, V_{1}\right)$ and $\mathrm{FK}_{\mathcal{R}}\left(\mathcal{P} ; \Psi^{\prime}\right)=\mathrm{FK}_{\mathcal{R}}^{s}\left(U_{1}^{\prime}, V_{1}^{\prime}\right)$, where $\left(U_{1}, V_{1}\right)$ and $\left(U_{1}^{\prime}, V_{1}^{\prime}\right)$ are $\mathrm{SL}_{\mathcal{P}}$-equivalences from $-\iota_{4 \mathbf{e}_{j}}\left(-\mathrm{B}_{E}\right)$ to $\mathrm{B}_{F}$ and from $\mathrm{B}_{E_{u,-}}$ to $\mathrm{B}_{F^{\prime}}$, respectively. The composition thus gives an $\mathrm{SL}_{\mathcal{P}}$-equivalence $\left(U^{\prime}, V^{\prime}\right)$ from $\mathrm{B}_{F}$ to $\mathrm{B}_{F^{\prime}}$.

It now follows from Boy02, Theorem 4.4] and Proposition 6.1 that there is a $\mathcal{P}$ equivariant isomorphism $\Phi^{\prime}$ from $C^{*}(F) \otimes \mathbb{K}$ to $C^{*}\left(F^{\prime}\right) \otimes \mathbb{K}$ such that $\operatorname{FK}_{\mathcal{R}}\left(\mathcal{P}, \Phi^{\prime}\right)=$ $\mathrm{FK}_{\mathcal{R}}^{s}\left(U^{\prime}, V^{\prime}\right)$. Then $\Phi:=\left(\Psi^{\prime}\right)^{-1} \circ \Phi^{\prime} \circ \Psi$ is a $\mathcal{P}$-equivariant isomorphism from $C^{*}(E) \otimes \mathbb{K}$ to $C^{*}\left(E_{u,--}\right) \otimes \mathbb{K}$ such that

$$
\operatorname{FK}_{\mathcal{R}}(\mathcal{P}, \Phi)=\operatorname{FK}_{\mathcal{R}}^{s}(U, V)
$$

The following theorem is the key result to prove that the Cuntz splice induces stably isomorphic graph $C^{*}$-algebras in a way where we can control the induced maps on the reduced filtered $K$-theory. Since it does not increase the difficulty of the proof, for the convenience of possible later application we state and prove the result in slightly greater generality than needed in this paper - allowing for infinite emitters and sinks. 
Theorem 7.3. Let $E$ be a graph with finitely many vertices, and let $u$ be a regular vertex in $E^{0}$. Assume that $\mathrm{B}_{E} \in \mathfrak{M}_{\mathcal{P}}^{\circ \circ}(\mathbf{m} \times \mathbf{n}, \mathbb{Z})$ and that $u$ belongs to the block $j \in \mathcal{P}$. Then there exist $U \in \mathrm{SL}_{\mathcal{P}}\left(\mathbf{m}+4 \mathbf{e}_{j}, \mathbb{Z}\right)$ and $V \in \mathrm{GL}_{\mathcal{P}}\left(\mathbf{n}+4 \mathbf{e}_{j}, \mathbb{Z}\right)$ that are the identity matrix everywhere except for the jth diagonal block, all the diagonal blocks of $U$ and $V$ have determinant 1 except for the $j$ th diagonal block of $V$, which has determinant -1 , and $(U, V)$ is a $\mathrm{GL}_{\mathcal{P}}$-equivalence from $-\iota_{2 \mathbf{e}_{j}}\left(-\mathrm{B}_{E_{u,-}}^{\bullet}\right)$ to $\mathrm{B}_{E_{u,--}}$. Moreover, there exists a $\mathcal{P}$-equivariant isomorphism $\Phi$ from $C^{*}\left(E_{u,-}\right)$ to $C^{*}\left(E_{u,--}\right)$ such that $\mathrm{FK}_{\mathcal{R}}(\mathcal{P} ; \Phi)=\operatorname{FK}_{\mathcal{R}}(U, V)$.

Proof. In the proof of ERRS16a we found two representations of $C^{*}\left(E_{u,-}\right)$ and $C^{*}\left(E_{u,--}\right)$ inside a $C^{*}$-algebra $\mathfrak{A}$, and showed that they are in fact equal. Now, we will produce $U$ and $V$ such that the identity isomorphism is exactly given by the isomorphism $\operatorname{FK}_{\mathcal{R}}(U, V)$ - when we use the canonical identifications of the $K$ theory. We refer the reader to the detailed definitions in the proof of [ERRS16a].

Let $U \in \mathrm{SL}_{\mathcal{P}}\left(\mathbf{m}+4 \mathbf{e}_{j}, \mathbb{Z}\right)$ and $V \in \operatorname{GL}_{\mathcal{P}}\left(\mathbf{n}+4 \mathbf{e}_{j}, \mathbb{Z}\right)$ be the identity matrix everywhere except for the $j$ th diagonal block where they are

$$
\begin{aligned}
& U_{0}=\left(\begin{array}{lll}
I & & 0 \\
0 & \left(\begin{array}{llll}
1 & 0 & 0 & 1 \\
0 & 1 & 0 & 0 \\
0 & 0 & 1 & 0 \\
0 & 0 & 0 & 1
\end{array}\right)
\end{array}\right), \\
& V_{0}=\left(\begin{array}{cccc}
I & & 0 & \\
0 & \left(\begin{array}{cccc}
1 & 0 & 0 & 0 \\
0 & 1 & 0 & 0 \\
-1 & 0 & 0 & -1 \\
0 & 0 & -1 & 0
\end{array}\right)
\end{array}\right),
\end{aligned}
$$

respectively. It is clear that all the diagonal blocks of $U$ and $V$ have determinant 1 except for the $j$ th diagonal block of $V$, which has determinant -1 . Moreover, it is easy to see that $(U, V)$ is a $\mathrm{GL}_{\mathcal{P}}$-equivalence from $-\iota_{2 \mathbf{e}_{j}}\left(-\mathrm{B}_{E_{u,-}}^{\bullet_{u}}\right)$ to $\mathrm{B}_{E_{u,--}}^{\boldsymbol{E}_{u}}$ (where we let the order of the added vertices follow the orders $v_{1}, v_{2}$ and $w_{1}, w_{2}, w_{3}, w_{4}$, respectively). We will show that the identity isomorphism is exactly given by the isomorphism $\operatorname{FK}_{\mathcal{R}}(U, V)$ - when we use the canonical identifications of the $K$ theory.

Recall from the proof of [ERRS16b, Theorem 4.5] that $\left\{q_{v}, t_{e}\right\}$ is the CuntzKrieger $E_{u,-}$-family in $\mathfrak{A}$ that was constructed and $\left\{P_{v}, S_{e}\right\}$ is the Cuntz-Krieger $E_{u,--}$-family in $\mathfrak{A}$ that was constructed, and that we call these two families $\mathcal{S}$ and $\mathcal{T}$, respectively. Moreover, since $E$ has finitely many vertices, these $C^{*}$-algebras are all unital sub- $C^{*}$-algebras.

It is clear that we have that $\left[q_{v_{1}}\right]_{0}=\left[P_{w_{1}}\right]_{0}=\left[P_{w_{3}}\right]_{0}=\left[P_{w_{4}}\right]_{0}=0$ and $\left[q_{v_{2}}\right]_{0}=$ $-\left[q_{u}\right]_{0}=-\left[P_{u}\right]_{0}=\left[P_{w_{2}}\right]_{0}$ in $K_{0}(\mathfrak{I})$ for any gauge invariant ideal $\mathfrak{I}$ containing $q_{v_{i}}$, $P_{w_{j}}, i=1,2, j=1,2,3,4$, and similarly for subquotients. Since we have $P_{v}=q_{v}$ for all $v \in E^{0}$, it now follows that this $\mathrm{GL}_{\mathcal{P}}$-equivalence induces exactly the desired maps on the $K_{0}$-groups.

Now we need to show that on the gauge simple subquotients, we get the right identification of $K_{1}$. For all subquotients except for the $j$ th, this is clear. For notational convenience, assume that $u$ corresponds to the last row of the diagonal block $\mathrm{B}_{E}^{\bullet}\{j\}$, i.e., the $m_{j}$ th row. We may also without loss of generality assume that $j$ is the only component of $E$. Now let $\mathbf{x}=\left(x_{i}\right)_{i=1}^{m_{j}+4} \in \operatorname{ker}\left(-\iota_{2 \mathbf{e}_{j}}\left(-\mathrm{B}_{E_{u,-}}\right)\right)^{\top}$. Note that $x_{m_{j}+1}=x_{m_{j}+3}=x_{m_{j}+4}=0$ and $x_{m_{j}}+x_{m_{j}+2}=0$. Note also that this implies that $\mathbf{x} \in \operatorname{ker}\left(\mathrm{B}_{E_{u,--}}^{\bullet}\right)^{\top}$. Now we construct the partial isometries $\mathrm{S}_{\mathbf{x}}$ 
and $\tilde{\mathrm{S}}_{\mathbf{x}}$, the projections $\mathrm{P}_{\mathbf{x}}=\mathrm{S}_{\mathbf{x}}^{*} \mathrm{~S}_{\mathbf{x}}=\mathrm{S}_{\mathbf{x}} \mathrm{S}_{\mathbf{x}}^{*}, \tilde{\mathrm{P}}_{\mathbf{x}}=\tilde{\mathrm{S}}_{\mathbf{x}}^{*} \tilde{\mathrm{S}}_{\mathbf{x}}=\tilde{\mathrm{S}}_{\mathbf{x}} \tilde{\mathrm{S}}_{\mathbf{x}}^{*}$ and the unitaries $\mathrm{U}_{\mathbf{x}}=\mathrm{S}_{\mathbf{x}}+1-\mathrm{P}_{\mathbf{x}}$ and $\tilde{\mathrm{U}}_{\mathbf{x}}=\tilde{\mathrm{S}}_{\mathbf{x}}+1-\tilde{\mathrm{P}}_{\mathbf{x}}$ in matrix algebras over $C^{*}(\mathcal{S})=C^{*}(\mathcal{T})$ as in CET12, where the ones without a tilde correspond to the family $\mathcal{S}$ while the ones with a tilde correspond to the family $\mathcal{T}$. From the matrix $\left(U_{0}^{\top}\right)^{-1}$ we see that what we need to show is that $\left[\mathrm{U}_{\mathbf{x}}\right]_{1}=\left[\tilde{\mathrm{U}}_{\mathbf{x}}\right]_{1}$.

In CET12 we have the following definitions:

$$
\begin{aligned}
& L_{v}^{+}=\left\{(e, i) \mid e \in E_{u,-}^{1}, r(e)=v, 1 \leq i \leq-x_{s(e)}\right\} \cup\left\{(v, i) \mid 1 \leq i \leq x_{v}\right\}, \\
& L_{v}^{-}=\left\{(e, i) \mid e \in E_{u,-}^{1}, r(e)=v, 1 \leq i \leq x_{s(e)}\right\} \cup\left\{(v, i) \mid 1 \leq i \leq-x_{v}\right\}, \\
& \tilde{L}_{w}^{+}=\left\{(f, k) \mid e \in E_{u,--}^{1}, r(e)=w, 1 \leq k \leq-x_{s(f)}\right\} \cup\left\{(w, k) \mid 1 \leq k \leq x_{w}\right\}, \\
& \tilde{L}_{w}^{-}=\left\{(f, k) \mid e \in E_{u,--}^{1}, r(e)=w, 1 \leq k \leq x_{s(e)}\right\} \cup\left\{(w, k) \mid 1 \leq k \leq-x_{w}\right\},
\end{aligned}
$$

for $v \in E_{u,-}^{0}$ and all $w \in E_{u,--}^{0}$. Since $x_{m_{j}+1}=x_{m_{j}+3}=x_{m_{j}+4}=0$, neither any of the edges $d_{2}, e_{1}, e_{2}, f_{1}, f_{2}, f_{5}, f_{6}, f_{7}, f_{8}, f_{9}, f_{10}$ nor any of the vertices $v_{1}, w_{1}$, $w_{3}, w_{4}$ will appear in any such pair. It is clear that $L_{v}^{+}=\tilde{L}_{v}^{+}$and $L_{v}^{-}=\tilde{L}_{v}^{-}$for all $v \in E^{0}$. Also we see that $L_{w_{i}}^{+}=\emptyset=\tilde{L}_{w_{i}}^{-}$for $i=3,4$. The substitutions $v_{2} \mapsto w_{2}$, $e_{3} \mapsto f_{3}$ and $e_{4} \mapsto f_{4}$ will define canonical bijections from $L_{v_{i}}^{+}$to $\tilde{L}_{w_{i}}^{+}$and from $L_{v_{i}}^{-}$ to $\tilde{L}_{w_{i}}^{-}$, for $i=1,2$.

Thus the size of $U_{\mathbf{x}}$ and $\tilde{U}_{\mathbf{x}}$ is the same. We want to show that $\left[U_{\mathbf{x}}\right]_{1}=\left[\tilde{U}_{\mathbf{x}}\right]_{1}$. Since $\left[\mathrm{U}_{-\mathbf{x}}\right]_{1}=-\left[\mathrm{U}_{\mathbf{x}}\right]_{1}$ and $\left[\tilde{U}_{-\mathbf{x}}\right]_{1}=-\left[\tilde{U}_{\mathbf{x}}\right]_{1}$, we may assume that $x_{u}=x_{m_{j}} \geq 0$. With a slight abuse of notation, we let $z$ denote the diagonal matrix of this matrix algebra having $z$ as all diagonal entries. Using the definitions and matrix units from CET12, we see that

$$
\begin{aligned}
z \mathrm{U}_{\mathbf{x}} z^{*}-\tilde{\mathrm{U}}_{\mathbf{x}}= & \sum_{1 \leq i \leq x_{u}}\left(\left(z t_{e_{3}}^{*} z^{*}-s_{f_{3}}^{*}\right) \mathrm{E}_{\left[e_{3}, i\right],\left\langle v_{2}, i\right\rangle}+\left(z t_{e_{4}}^{*} z^{*}-s_{f_{4}}^{*}\right) \mathrm{E}_{\left[e_{4}, i\right],\left\langle v_{2}, i\right\rangle}\right. \\
& \left.+\left(P_{w_{2}}-z q_{v_{2}} z^{*}\right) \mathrm{E}_{\left\langle v_{2}, i\right\rangle,\left\langle v_{2}, i\right\rangle}\right) .
\end{aligned}
$$

Now we see that

$$
\begin{aligned}
& z \mathrm{U}_{\mathbf{x}} z^{*} \tilde{\mathrm{U}}_{\mathbf{x}}^{*}=1+\left(z \mathrm{U}_{\mathbf{x}} z^{*}-\tilde{\mathrm{U}}_{\mathbf{x}}\right) \tilde{\mathrm{U}}_{\mathbf{x}}^{*} \\
&=1+\sum_{1 \leq i \leq x_{u}}\left(\sum _ { j = 3 } ^ { 4 } \left(\left(z t_{e_{j}}^{*} z^{*}-z t_{e_{j}}^{*} z^{*} P_{w_{2}}\right) \mathrm{E}_{\left[e_{j}, i\right],\left\langle v_{2}, i,\right\rangle}\right.\right. \\
&\left.\left.\quad+\sum_{k=3}^{4}\left(z t_{e_{j}}^{*} z^{*}-s_{e_{j}}^{*}\right) s_{e_{k}} \mathrm{E}_{\left[e_{j}, i\right],\left[e_{k}, i\right]}\right)\right) .
\end{aligned}
$$

Note that this is in fact a unitary in $M_{h}(\mathfrak{A})$ of the form $1_{M_{h}\left(\mathfrak{A}_{0}\right)}+U$ where $U$ is the unitary

$$
\begin{aligned}
U=1_{M_{h}(\mathcal{E})}+\sum_{1 \leq i \leq x_{u}}\left(\sum _ { j = 3 } ^ { 4 } \left(\left(z t_{e_{j}}^{*} z^{*}-z t_{e_{j}}^{*} z^{*} P_{w_{2}}\right) \mathrm{E}_{\left[e_{j}, i\right],\left\langle v_{2}, i,\right\rangle}\right.\right. \\
\left.\left.+\sum_{k=3}^{4}\left(z t_{e_{j}}^{*} z^{*}-s_{e_{j}}^{*}\right) s_{e_{k}} \mathrm{E}_{\left[e_{j}, i\right],\left[e_{k}, i\right]}\right)\right)
\end{aligned}
$$


in $M_{h}(\mathcal{E})$ and $h$ is the number of elements in $\mathrm{L}_{\mathbf{x}}^{+}$. Since $K_{1}(\mathcal{E})=0$ it follows from the split exactness of $K_{1}$ that $\left[z \mathrm{U}_{\mathbf{x}} z^{*} \tilde{U}_{\mathbf{x}}^{*}\right]_{1}=0$ in $K_{1}(\mathfrak{A})$. Since the embedding of $\mathfrak{A}$ into $C^{*}(\mathcal{S})=C^{*}(\mathcal{T})$ is unital, it follows that $\left[z \mathrm{U}_{\mathbf{x}} z^{*} \tilde{\mathrm{U}}_{\mathbf{x}}^{*}\right]_{1}=0$ in $K_{1}\left(C^{*}(\mathcal{S})\right)$. Thus $\left[\mathrm{U}_{\mathbf{x}}\right]_{1}=\left[\tilde{\mathrm{U}}_{\mathbf{x}}\right]_{1}$ in $K_{1}\left(C^{*}(\mathcal{S})\right)$.

By combining the above, we get the following important result.

Corollary 7.4. Let $E$ be a finite graph with no sources and no sinks satisfying Condition $(\mathrm{K})$ such that $\mathrm{B}_{E} \in \mathfrak{M}_{\mathcal{P}}^{+}(\mathbf{n}, \mathbb{Z})$. Let $u$ be a vertex in $E^{0}$ and assume that $u$ belongs to the block $j \in \mathcal{P}$. Then $\mathrm{B}_{E_{u,-}} \in \mathfrak{M}_{\mathcal{P}}^{\circ \circ}\left(\mathbf{n}+2 \mathbf{e}_{j}, \mathbb{Z}\right)$, and there exist $U \in \mathrm{SL}_{\mathcal{P}}\left(\mathbf{n}+2 \mathbf{e}_{j}, \mathbb{Z}\right)$ and $V \in \mathrm{GL}_{\mathcal{P}}\left(\mathbf{n}+2 \mathbf{e}_{j}, \mathbb{Z}\right)$ such that the following holds. The matrices $U$ and $V$ are equal to the identity matrices everywhere except for the $j$ th diagonal block. All the diagonal blocks of $U$ and $V$ have determinant 1 except for the $j$ th diagonal block of $V$, which has determinant -1 and $(U, V)$ is a $\mathrm{GL}_{\mathcal{P}}$-equivalence from $-\iota_{2 \mathbf{e}_{j}}\left(-\mathrm{B}_{E}\right)$ to $\mathrm{B}_{E_{u,-}}$. Moreover, there exists a $\mathcal{P}$-equivariant isomorphism $\Phi: C^{*}(E) \otimes \mathbb{K} \rightarrow C^{*}\left(E_{u,-}\right) \otimes \mathbb{K}$ such that $\mathrm{FK}_{\mathcal{R}}(\mathcal{P} ; \Phi)=\mathrm{FK}_{\mathcal{R}}^{s}(U, V)$.

Proof. It follows from Theorem 7.3 and Proposition 7.2 that the statement holds if we say that the $\mathcal{P}$-equivariant isomorphism $\Phi$ is induced by such a $\mathrm{GL}_{\mathcal{P}}$-equivalence from $-\iota_{4 \mathbf{e}_{j}}\left(-\mathrm{B}_{E}\right)$ to $-\iota_{2 \mathbf{e}_{j}}\left(-\mathrm{B}_{E_{u,-}}\right)$. Let $\left(U_{1}, V_{1}\right)$ be the $\mathrm{SL}_{\mathcal{P}}$-equivalence from the proof of Proposition 7.2 and let $\left(U_{2}, V_{2}\right)$ be the $\mathrm{GL}_{\mathcal{P}}$-equivalence from the proof of Theorem 7.3. By computing $U_{2}^{-1} U_{1}$ and $V_{1} V_{2}^{-1}$ we see that these are in fact of the form $\iota_{2 \mathbf{e}_{j}}(U)$ and $\iota_{2 \mathbf{e}_{j}}(V)$, respectively, for matrices $U \in \operatorname{SL}_{\mathcal{P}}\left(\mathbf{n}+2 \mathbf{e}_{j}, \mathbb{Z}\right)$ and $V \in \mathrm{GL}_{\mathcal{P}}\left(\mathbf{n}+2 \mathbf{e}_{j}, \mathbb{Z}\right)$ of the desired form.

Theorem 7.5. Let $A$ and $A^{\prime}$ be nondegenerate $\{0,1\}$-matrices satisfying Condition (II). Let $X=\operatorname{Prim}\left(\mathcal{O}_{A}\right)$ and assume that there is a homeomorphism between $\operatorname{Prim}\left(\mathcal{O}_{A}\right)$ and $\operatorname{Prim}\left(\mathcal{O}_{A^{\prime}}\right)$, and use this homeomorphism to view $\mathcal{O}_{A} \otimes \mathbb{K}$ and $\mathcal{O}_{A^{\prime}} \otimes \mathbb{K}$ as $X$-algebras. Assume that $\varphi: \mathrm{FK}_{\mathcal{R}}\left(X ; \mathcal{O}_{A} \otimes \mathbb{K}\right) \rightarrow \mathrm{FK}_{\mathcal{R}}\left(X ; \mathcal{O}_{A^{\prime}} \otimes \mathbb{K}\right)$ is an isomorphism. Then there exists an $X$-equivariant isomorphism $\Phi: \mathcal{O}_{A} \otimes \mathbb{K} \rightarrow$ $\mathcal{O}_{A^{\prime}} \otimes \mathbb{K}$ such that $\mathrm{FK}_{\mathcal{R}}(X ; \Phi)=\varphi$.

Proof.

Step 1. According to Lemma 6.3 we may consider two finite graphs $E$ and $F$ with no sinks and no sources satisfying Condition $(K)$ such that $\mathrm{B}_{E} \in \mathfrak{M}_{\mathcal{P}}^{+}\left(\mathbf{n}_{E}, \mathbb{Z}\right)$ and $\mathrm{B}_{F} \in \mathfrak{M}_{\mathcal{P}}^{+}\left(\mathbf{n}_{F}, \mathbb{Z}\right)$ and then prove the corresponding result for the graph $C^{*}$-algebras $C^{*}(E)$ and $C^{*}(F)$. We choose the block structure in such a way that it is over the same poset $\mathcal{P}$, and such that the block structures are compatible with the homeomorphism between $\operatorname{Prim}\left(\mathcal{O}_{A}\right)$ and $\operatorname{Prim}\left(\mathcal{O}_{A^{\prime}}\right) ; c f$. the discussion right after ERRS16b, Definition 4.15].

Step 2. According to Lemma 6.4 we may, moreover, assume that $A_{E}$ and $A_{F}$ have the same block structure. So we write $B_{E}, B_{F} \in \mathfrak{M}_{\mathcal{P}}^{+}(\mathbf{n}, \mathbb{Z})$, for some multi-index $\mathbf{n}$.

Step 3. It follows from [ERRS16b, Section 4.4] and Corollary 4.3 that there exists a $\operatorname{GL}_{\mathcal{P}}$-equivalence $(U, V)$ from $\mathrm{B}_{E}$ to $\mathrm{B}_{F}$ such that $\varphi=\mathrm{FK}_{\mathcal{R}}^{s}(U, V)$.

Step 4. For each $i \in \mathcal{P}$ with $\operatorname{det}(U\{i\}) \neq \operatorname{det}(V\{i\})$, we do as follows. Assume that $j \in \mathcal{P}$ with $\operatorname{det}(U\{j\}) \neq \operatorname{det}(V\{j\})$. Let $v$ be a vertex in the component corresponding to $j$. Cuntz-splice the graph $E$ at vertex $v$, and call the new graph $E_{1}$. 
Then $\mathrm{B}_{E_{1}} \in \mathfrak{M}_{\mathcal{P}}\left(\mathbf{n}+2 \mathbf{e}_{j}, \mathbb{Z}\right)$. Corollary 7.4 gives us an $X$-equivariant isomorphism $\Phi_{1}$ from $C^{*}\left(E_{1}\right) \otimes \mathbb{K}$ to $C^{*}(E) \otimes \mathbb{K}$ and a $\operatorname{GL}_{\mathcal{P}}$-equivalence $\left(U_{1}, V_{1}\right)$ from $\mathrm{B}_{E_{1}}$ to $-\iota_{2 \mathbf{e}_{j}}\left(-\mathrm{B}_{E}\right)$ such that $\mathrm{FK}_{\mathcal{R}}\left(X ; \Phi_{1}\right)=\mathrm{FK}_{\mathcal{R}}^{s}\left(U_{1}, V_{1}\right), \operatorname{det}\left(U_{1}\{j\}\right) \neq \operatorname{det}\left(V_{1}\{j\}\right)$ and $\operatorname{det}\left(U_{1}\{j\}\right)=\operatorname{det}\left(V_{1}\{j\}\right)=1$, for all $i \neq j$. By composing the GL P $_{\mathcal{P}}$-equivalences $\left(U_{1}, V_{1}\right)$ and $(U, V)$ and using column and row additions together with Proposition 6.1 in the same way as in the proof of Lemma 6.4, we see that we may without loss of generality assume that $\operatorname{det}(U\{i\})=\operatorname{det}(V\{i\})$, for all $i \in \mathcal{P}$.

Step 5. Consequently, in addition to the assumptions on $E$ and $F$, we now assume that $\operatorname{det}(U\{i\})=\operatorname{det}(V\{i\})$, for all $i \in \mathcal{P}$. Let $\mathbf{r}$ be the multi-index where all entries are 2. Now let $U^{\prime}, V^{\prime} \in \mathrm{SL}_{\mathcal{P}}(\mathbf{n}+\mathbf{r}, \mathbb{Z})$ be given as follows. We obtain $U^{\prime}$ from $\iota_{\mathbf{r}}(U)$ by interchanging the last two rows of each row-block $i$ that satisfies $\operatorname{det}(U\{i\})=-1$, and we similarly obtain $V^{\prime}$ from $\iota_{\mathbf{r}}(V)$ by interchanging the last two rows of each row-block $i$ that satisfies $\operatorname{det}(V\{i\})=-1$. It is now clear that $\left(U^{\prime}, V^{\prime}\right)$ is an $\mathrm{SL}_{\mathcal{P}^{-}}$ equivalence from $-\iota_{\mathbf{r}}\left(-\mathrm{B}_{E}\right)$ to $-\iota_{\mathbf{r}}\left(-\mathrm{B}_{F}\right)$ and it is also clear that $\mathrm{FK}_{\mathcal{R}}^{s}(U, V)=$ $\mathrm{FK}_{\mathcal{R}}^{s}\left(U^{\prime}, V^{\prime}\right)$. By using column and row additions in the same way as in the proof of Lemma 6.4 together with Proposition 6.1 we see that we get two finite graphs $E^{\prime}$ and $F^{\prime}$ satisfying Condition $(\mathrm{K})$ with no sinks and no sources such that $\mathrm{B}_{E^{\prime}}, \mathrm{B}_{F^{\prime}} \in$ $\mathfrak{M}_{\mathcal{P}}^{+}(\mathbf{n}+\mathbf{r}, \mathbb{Z})$ and such that there exist $\mathcal{P}$-equivariant isomorphisms $\Psi$ from $C^{*}(E) \otimes$ $\mathbb{K}$ to $C^{*}\left(E^{\prime}\right) \otimes \mathbb{K}$ and $\Psi^{\prime}$ from $C^{*}(F) \otimes \mathbb{K}$ to $C^{*}\left(F^{\prime}\right) \otimes \mathbb{K}$ with $\mathrm{FK}_{\mathcal{R}}(\mathcal{P} ; \Psi)=$ $\operatorname{FK}_{\mathcal{R}}^{s}\left(U_{1}, V_{1}\right)$ and $\mathrm{FK}_{\mathcal{R}}\left(\mathcal{P} ; \Psi^{\prime}\right)=\mathrm{FK}_{\mathcal{R}}^{s}\left(U_{2}, V_{2}\right)$, where $\left(U_{1}, V_{1}\right)$ and $\left(U_{2}, V_{2}\right)$ are $\mathrm{SL}_{\mathcal{P}^{-}}$ equivalences from $-\iota_{\mathbf{r}}\left(-\mathrm{B}_{E}\right)$ to $\mathrm{B}_{E^{\prime}}$ and from $-\iota_{\mathbf{r}}\left(-\mathrm{B}_{F}\right)$ to $\mathrm{B}_{F^{\prime}}$, respectively. Thus by composition, the problem is reduced to lifting the filtered $K$-theory isomorphism $\operatorname{FK}_{\mathcal{R}}^{s}(\tilde{U}, \tilde{V})$ induced by an $\mathrm{SL}_{\mathcal{P}}$-equivalence $(\tilde{U}, \tilde{V})=\left(U_{2} U^{\prime} U_{1}^{-1}, V_{1}^{-1} V^{\prime} V_{2}\right)$ from $\mathrm{B}_{F}$ to $\mathrm{B}_{F^{\prime}}$.

Step 6. Now the theorem follows from Proposition 6.1 and Boy02, Theorem 4.4].

We now show how Theorem 7.5 can be used to prove Theorem 3.1 .

Proof of Theorem 3.1. Let $A$ and $A^{\prime}$ be nondegenerate matrices with entries from $\{0,1\}$ satisfying Condition (II). Suppose that there exists an isomorphism $(\kappa, \rho)$ from $\mathrm{FK}_{\text {red }}\left(\mathcal{O}_{A}\right)$ to $\mathrm{FK}_{\text {red }}\left(\mathcal{O}_{A^{\prime}}\right)$. Since $\rho$ is a homeomorphism from $\operatorname{Prim}\left(\mathcal{O}_{A}\right)$ to $\operatorname{Prim}\left(\mathcal{O}_{A^{\prime}}\right)$, it follows that $\left(\mathcal{O}_{A^{\prime}}, \rho^{-1}\right)$ is a $C^{*}$-algebra over $\operatorname{Prim}\left(\mathcal{O}_{A}\right)$ and that we can consider $\kappa$ as an isomorphism from $\operatorname{FK}_{\mathcal{R}}\left(\operatorname{Prim}\left(\mathcal{O}_{A}\right) ; \mathcal{O}_{A}\right)$ to $\mathrm{FK}_{\mathcal{R}}\left(\operatorname{Prim}\left(\mathcal{O}_{A}\right) ; \mathcal{O}_{A^{\prime}}\right)$. Thus, it follows from Theorem 7.5 that there exists a $\operatorname{Prim}\left(\mathcal{O}_{A}\right)$-equivariant isomorphism $\Psi: \mathcal{O}_{A} \otimes \mathbb{K} \rightarrow \mathcal{O}_{A^{\prime}} \otimes \mathbb{K}$ such that $\mathrm{FK}_{\mathcal{R}}\left(\operatorname{Prim}\left(\mathcal{O}_{A}\right) ; \Psi\right)=\kappa$ up to canonical identification. By construction, $\Psi$ induces $\rho$. Hence, $\Psi: \mathcal{O}_{A} \otimes \mathbb{K} \rightarrow \mathcal{O}_{A^{\prime}} \otimes \mathbb{K}$ is an isomorphism such that $\mathrm{FK}_{\text {red }}^{s}(\Psi)=(\kappa, \rho)$.

Theorem 7.6. Let $A$ and $A^{\prime}$ be nondegenerate $\{0,1\}$-matrices satisfying Condition (II). Let $X=\operatorname{Prim}\left(\mathcal{O}_{A}\right)$ and assume that there is a homeomorphism between $\operatorname{Prim}\left(\mathcal{O}_{A}\right)$ and $\operatorname{Prim}\left(\mathcal{O}_{A^{\prime}}\right)$, and use this homeomorphism to view $\mathcal{O}_{A}$ and $\mathcal{O}_{A^{\prime}}$ as $X$-algebras. Assume that $\varphi: \mathrm{FK}_{\mathcal{R}}\left(X ; \mathcal{O}_{A}\right) \rightarrow \mathrm{FK}_{\mathcal{R}}\left(X ; \mathcal{O}_{A^{\prime}}\right)$ is an isomorphism that preserves the class of the unit in $K_{0}$. Then there exists an $X$-equivariant isomorphism $\Phi: \mathcal{O}_{A} \rightarrow \mathcal{O}_{A^{\prime}}$ such that $\operatorname{FK}_{\mathcal{R}}(X ; \Phi)=\varphi$.

Proof. This follows from Definition 2.2, Theorem 17.5, ABK14b, Lemma 8.3 and Definition 8.4], and [ERR13c, Theorem 3.3] (by [AMP07, Corollary 7.2], row-finite graph $C^{*}$-algebras have the stable weak cancellation property). 
Proof of Theorem 3.2. This can be proved in a similar fashion to how Theorem 3.1 was proved, using Theorem 7.6 instead of Theorem 7.5 .

\section{ACKNOWLEDGEMENTS}

This work was partially supported by a grant from the Simons Foundation (No. 279369 to the third-named author). Some of the work was conducted while all three authors were attending the research program Classification of operator algebras: Complexity, rigidity, and dynamics at the Mittag-Leffler Institute, January-April 2016. We thank the institute and its staff for the excellent work conditions provided. The second-named author thanks the University of Hawaii, Hilo for their hospitality during a visit, where part of this work was done. The publication of this paper was supported by the Faroese Research Council. The authors wish to express their gratitude for this support.

\section{RefERENCES}

[ABK14a] Sara E. Arklint, Rasmus Bentmann, and Takeshi Katsura, The K-theoretical range of Cuntz-Krieger algebras, J. Funct. Anal. 266 (2014), no. 8, 5448-5466, DOI 10.1016/j.jfa.2014.01.020. MR.3177344

[ABK14b] Sara E. Arklint, Rasmus Bentmann, and Takeshi Katsura, Reduction of filtered Ktheory and a characterization of Cuntz-Krieger algebras, J. K-Theory 14 (2014), no. 3, 570-613, DOI 10.1017/is014009013jkt281. MR3349327

[AMP07] Pere Ara, Maria Angeles Moreno, and Enrique Pardo, Nonstable K-theory for graph algebras, Algebr. Represent. Theory 10 (2007), no. 2, 157-178, DOI 10.1007/s10468006-9044-z. MR2310414

[ARR12] Sara E. Arklint, Gunnar Restorff, and Efren Ruiz, Filtrated K-theory for real rank zero $C^{*}$-algebras, Internat. J. Math. 23 (2012), no. 8, 1250078, 19, DOI 10.1142/S0129167X12500784. MR2949216

[ARR16] Sara E. Arklint, Gunnar Restorff, and Efren Ruiz, Classification of real rank zero, purely infinite $C^{*}$-algebras with at most four primitive ideals, J. Funct. Anal. 271 (2016), no. 7, 1921-1947, DOI 10.1016/j.jfa.2016.05.014. MR3535322

[BH03] Mike Boyle and Danrun Huang, Poset block equivalence of integral matrices, Trans. Amer. Math. Soc. 355 (2003), no. 10, 3861-3886, DOI 10.1090/S0002-9947-03-029477. MR 1990568

[BHRS02] Teresa Bates, Jeong Hee Hong, Iain Raeburn, and Wojciech Szymański, The ideal structure of the $C^{*}$-algebras of infinite graphs, Illinois J. Math. 46 (2002), no. 4, 1159-1176. MR 1988256

[BK11] Rasmus Bentmann and Manuel Köhler, Universal coefficient theorems for $C^{*}$-algebras over finite topological spaces, arXiv e-prints (2011), arXiv:1101.5702v3.

[Bon04] Alexander Bonkat, Bivariante K-Theorie für Kategorien projektiver Systeme von $C^{*}$-Algebren, Ph.D. thesis, Westfälischen Wilhelms-Universität Münster, Jan 2004, Published in Preprintreihe SFB 478 Geometrische Strukturen in der Mathematik, Heft 319.

[Boy02] Mike Boyle, Flow equivalence of shifts of finite type via positive factorizations, Pacific J. Math. 204 (2002), no. 2, 273-317, DOI 10.2140/pjm.2002.204.273. MR.1907894

[Bro77] Lawrence G. Brown, Stable isomorphism of hereditary subalgebras of $C^{*}$-algebras, Pacific J. Math. 71 (1977), no. 2, 335-348. MR0454645

[CET12] Toke Meier Carlsen, Søren Eilers, and Mark Tomforde, Index maps in the K-theory of graph algebras, J. K-Theory 9 (2012), no. 2, 385-406, DOI 10.1017/is011004017jkt156. MR2922394

[Cun81] Joachim Cuntz, A class of $C^{*}$-algebras and topological Markov chains. II. Reducible chains and the Ext-functor for $C^{*}$-algebras, Invent. Math. 63 (1981), no. 1, 25-40, DOI 10.1007/BF01389192. MR608527 
[ER06] Søren Eilers and Gunnar Restorff, On Rørdam's classification of certain $C^{*}$-algebras with one non-trivial ideal, Operator Algebras: The Abel Symposium 2004, Abel Symp., vol. 1, Springer, Berlin, 2006, pp. 87-96, DOI 10.1007/978-3-540-34197-0_4. MR2265044

[ERR09] Søren Eilers, Gunnar Restorff, and Efren Ruiz, Classification of extensions of classifiable $C^{*}$-algebras, Adv. Math. 222 (2009), no. 6, 2153-2172, DOI 10.1016/j.aim.2009.07.014. MR 2562779

[ERR13a] Søren Eilers, Gunnar Restorff, and Efren Ruiz, Classification of graph $C^{*}$-algebras with no more than four primitive ideals, Operator algebra and dynamics, Springer Proc. Math. Stat., vol. 58, Springer, Heidelberg, 2013, pp. 89-129, DOI 10.1007/9783-642-39459-1_5. MR3142033

[ERR13b] Søren Eilers, Gunnar Restorff, and Efren Ruiz, Classifying $C^{*}$-algebras with both finite and infinite subquotients, J. Funct. Anal. 265 (2013), no. 3, 449-468, DOI 10.1016/j.jfa.2013.05.006. MR3056712

[ERR13c] Søren Eilers, Gunnar Restorff, and Efren Ruiz, Strong classification of extensions of classifiable $C^{*}$-algebras, arXiv e-prints (2013), arXiv:1301.7695v1.

[ERRS16a] Søren Eilers, Gunnar Restorff, Efren Ruiz, and Adam P. W. Sørensen, The complete classification of unital graph $C^{*}$-algebras: Geometric and strong, arXiv e-prints (2016), arXiv: 1611.07120v1.

[ERRS16b] Søren Eilers, Gunnar Restorff, Efren Ruiz, and Adam P. W. Sørensen, Geometric classification of graph $C^{*}$-algebras over finite graphs, arXiv e-prints (2016), arXiv: $1604.05439 \mathrm{v} 1$.

[ERRS16c] Søren Eilers, Gunnar Restorff, Efren Ruiz, and Adam P. W. Sørensen, Invariance of the Cuntz splice, arXiv e-prints (2016), arXiv:1602.03709v2.

[ET10] Søren Eilers and Mark Tomforde, On the classification of nonsimple graph $C^{*}$ algebras, Math. Ann. 346 (2010), no. 2, 393-418, DOI 10.1007/s00208-009-0403-z. MR 2563693

[Fra84] John Franks, Flow equivalence of subshifts of finite type, Ergodic Theory Dynam. Systems 4 (1984), no. 1, 53-66, DOI 10.1017/S0143385700002261. MR758893

[Hua94] Danrung Huang, Flow equivalence of reducible shifts of finite type, Ergodic Theory Dynam. Systems 14 (1994), no. 4, 695-720, DOI 10.1017/S0143385700008129. MR.1304139

[Hua95] Danrun Huang, Flow equivalence of reducible shifts of finite type and Cuntz-Krieger algebras, J. Reine Angew. Math. 462 (1995), 185-217, DOI 10.1515/crll.1995.462.185. MR.1329907

[Hua96] Danrun Huang, The classification of two-component Cuntz-Krieger algebras, Proc. Amer. Math. Soc. 124 (1996), no. 2, 505-512, DOI 10.1090/S0002-9939-96-03079-1. MR.1301504

[Kir00] Eberhard Kirchberg, Das nicht-kommutative Michael-Auswahlprinzip und die Klassifikation nicht-einfacher Algebren (German, with English summary), $C^{*}$-algebras (Münster, 1999), Springer, Berlin, 2000, pp. 92-141. MR.1796912

[MN12] Ralf Meyer and Ryszard Nest, $\mathrm{C}^{*}$-algebras over topological spaces: filtrated Ktheory, Canad. J. Math. 64 (2012), no. 2, 368-408, DOI 10.4153/CJM-2011-061-x. MR2953205

[Phi00] N. Christopher Phillips, A classification theorem for nuclear purely infinite simple $C^{*}$-algebras, Doc. Math. 5 (2000), 49-114. MR1745197

[Res06] Gunnar Restorff, Classification of Cuntz-Krieger algebras up to stable isomorphism, J. Reine Angew. Math. 598 (2006), 185-210, DOI 10.1515/CRELLE.2006.074. MR2270572

[Res08] Gunnar Restorff, Classification of non-simple $C^{*}$-algebras, Ph.D. thesis, University of Copenhagen, 2008, URL: http://www.math.ku.dk/noter/filer/phd08gr.pdf.

[Ror95] Mikael Rørdam, Classification of Cuntz-Krieger algebras, K-Theory 9 (1995), no. 1, 31-58, DOI 10.1007/BF00965458. MR1340839

[Ror97] Mikael Rørdam, Classification of extensions of certain $C^{*}$-algebras by their six term exact sequences in K-theory, Math. Ann. 308 (1997), no. 1, 93-117, DOI $10.1007 / \mathrm{s} 002080050067 . \mathrm{MR} 1446202$ 
[RR07] Gunnar Restorff and Efren Ruiz, On Rørdam's classification of certain $C^{*}$-algebras with one non-trivial ideal. II, Math. Scand. 101 (2007), no. 2, 280-292, DOI 10.7146/math.scand.a-15045. MR2379290

[Szy02] Wojciech Szymański, General Cuntz-Krieger uniqueness theorem, Internat. J. Math. 13 (2002), no. 5, 549-555, DOI 10.1142/S0129167X0200137X. MR1914564

Department of Science and Technology, University of the Faroe Islands, Nóatún 3, FO-100 Tórshavn, the Faroe Islands

E-mail address: tokemc@setur.fo

Department of Science and Technology, University of the Faroe Islands, Nóatún 3, FO-100 Tórshavn, the Faroe Islands

E-mail address: gunnarr@setur.fo

Department of Mathematics, University of Hawail, Hilo, 200 W. Kawili St., Hilo, HAWAII 96720-4091

E-mail address: ruize@hawaii.edu 